\title{
Comparison of chestnut canker treatment procedures for hypovirus introduction
}

\author{
Brian C. Bell \\ West Virginia University
}

Follow this and additional works at: https://researchrepository.wvu.edu/etd

\section{Recommended Citation}

Bell, Brian C., "Comparison of chestnut canker treatment procedures for hypovirus introduction" (2004). Graduate Theses, Dissertations, and Problem Reports. 2023.

https://researchrepository.wvu.edu/etd/2023

This Thesis is protected by copyright and/or related rights. It has been brought to you by the The Research Repository @ WVU with permission from the rights-holder(s). You are free to use this Thesis in any way that is permitted by the copyright and related rights legislation that applies to your use. For other uses you must obtain permission from the rights-holder(s) directly, unless additional rights are indicated by a Creative Commons license in the record and/ or on the work itself. This Thesis has been accepted for inclusion in WVU Graduate Theses, Dissertations, and Problem Reports collection by an authorized administrator of The Research Repository @ WVU. For more information, please contact researchrepository@mail.wvu.edu. 
Comparison of chestnut canker treatment procedures for hypovirus introduction

Brian C. Bell

Thesis submitted to the Davis College of Agriculture, Forestry and Consumer Sciences at West Virginia University in partial fulfillment of the requirements for the degree of

\author{
Master of Science \\ in \\ Plant Pathology
}
William L. MacDonald, Ph.D., Chair
John R. Brooks, Ph.D.
James B. Kotcon, Ph.D.
Daniel G. Panaccione, Ph.D.

Division of Plant and Soil Sciences
Morgantown, West Virginia 2004

Keywords: hypovirulence, biocontrol, chestnut blight, Cryphonectria parasitica Copyright 2004 Brian C. Bell 


\begin{abstract}
Comparison of chestnut canker treatment procedures for hypovirus introduction
\end{abstract}

Brian C. Bell

Historically, hypovirulent strains of Cryphonectria parasitica have been introduced into cankers principally by inoculating wounds made to the margin of cankers. Other techniques have not been investigated extensively to determine if the method of canker treatment can play a role in the success of hypovirus transmission and thus biological control. To address this issue, twelve hypovirus introduction methods were tested. Seventy-two trees were inoculated in June 2002 with an orange-pigmented virulent strain of $C$. parasitica (two inoculations per tree). After 11 weeks, the resulting cankers were treated with a brown-pigmented hypovirulent $C$. parasitica isolate using one of the following methods: 1) a non-invasive treatment where the inoculum was painted onto the canker surface; 2) an invasive treatment where the canker face was wounded with a sharp blade prior to the painting application of the hypoviruses; and, 3) a margin punch treatment where a series of wounds were made around the canker perimeter and filled with inoculum of the hypovirulent strain. Additionally, half the cankers in each treatment were covered to keep treatment inoculum moist and to protect treatment inoculum from biotic and abiotic factors. Because one major barrier to hypovirus transmission is a system of vegetative incompatibility, isolates that were vegetatively compatible or incompatible with the canker inciting strain were employed. Hypovirus transmission was evaluated in November 2002 and May 2003 by culturing bark plug samples and evaluating the pigmentation and morphologies of isolates recovered. Canker dimensions also were recorded to determine if hypovirus transmission had an affect on canker expansion. When successful transmission was indicated, lightly pigmented orange hypovirulent isolates were recovered. When hypovirus transmission did not occur, virulent orange isolates were recovered; the recovery of brown-pigmented isolates indicated the recovery of the treatment inoculum. Recovery of orange-pigmented hypovirulent isolates was greatest when cankers were wounded. Punch wounded cankers resulted in significantly better recovery of orange-hypovirulent isolates than scratch wounded cankers. Likewise, cankers treated with compatible hypovirulent inoculum yielded more orange-pigmented hypovirulent isolates than cankers treated with incompatible hypovirulent inoculum. The brown-hypovirulent treatment inoculum persisted best when cankers were treated with incompatible inoculum; additionally, covering cankers significantly increased the recovery of the treatment inoculum. Cankers expanded significantly more when they were treated with incompatible inoculum compared to treatment with compatible inoculum. This study showed that wounding cankers prior to the application of hypovirulent treatment inoculum was paramount to an increase in the recovery of orange-pigmented hypovirulent isolates. Also, the use of treatment isolates that were vegetatively compatible with the canker inciting strain was found to be equally important. If survival of the treatment inoculum is a priority, canker coverings should be employed. However, these coverings had no apparent effect on the recovery of orange-hypovirulent isolates. 


\section{Acknowledgements}

The completion of this thesis has been a collaborative effort among many people. First and foremost, I would like to thank my advisor Dr. William MacDonald. Not only did he give me the chance to study under him, but his patience guided this project through to the very end. He also provided me with wisdom and insight about forest pathology issues. I also would like to thank my graduate committee members; Drs. Dan Panaccione, Jim Kotcon and John Brooks for their support and assistance with this project. Many thanks are due Mark Double for his guidance and support, not only in teaching me laboratory techniques, but also his assistance with field work and his great sense of humor. I also owe Jessica Bright enormous thanks for her assistance in processing lab samples and fieldwork. Without her, completion of this project would have been extended another year. I would also like to thank fellow graduate student Jenise Bauman for her assistance on those extra long days of fieldwork. Special thanks go out to the other graduate students with whom I shared my tenure at WVU. They provided me with friendship and encouragement throughout my graduate studies. I would like to thank the research division of MeadWestvaco for providing a field site on which this study was conducted. Thanks to Dr. George Seidal for assistance with all the statistical analyses conducted on data from this study. Finally, my family deserves my deepest gratitude. My parents, Barbara and Jack Bell, my grandparents, Marietta and Heath Bell, and my brother Jack have provided me understanding and support, not only through my graduate work, but throughout my life. 
Table of Contents

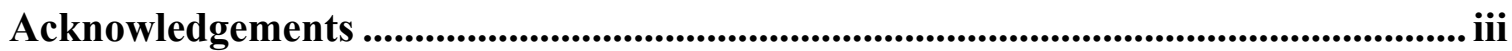

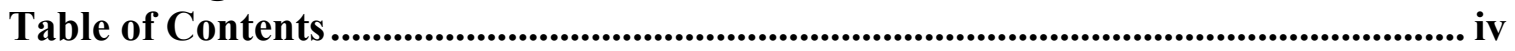

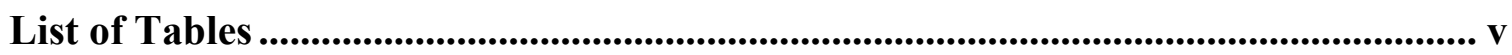

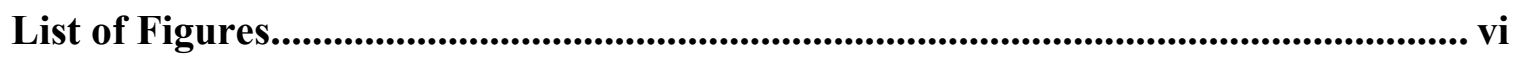

List of Appendices............................................................................................................................. viii

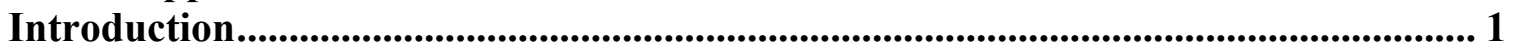

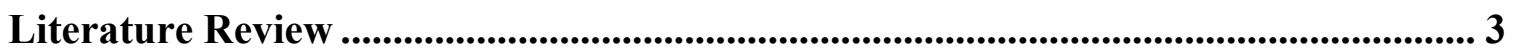

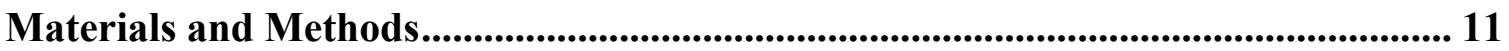

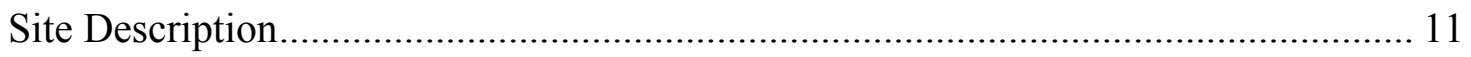

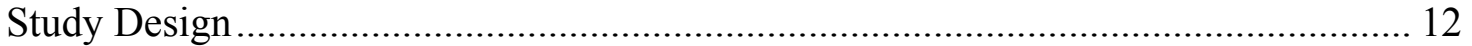

Isolate Selection .............................................................................................. 15

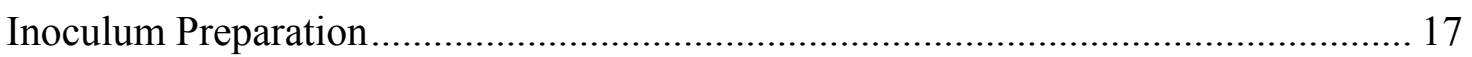

Canker Measurement and Sampling ................................................................. 18

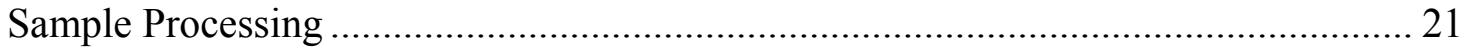

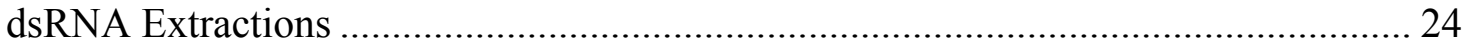

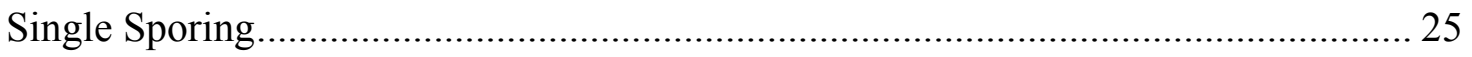

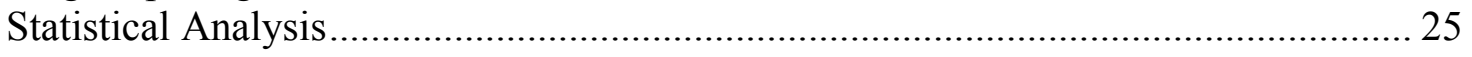

Results ............................................................................................................................................. 27

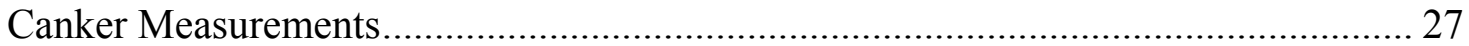

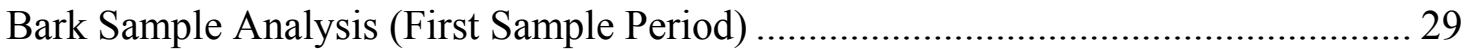

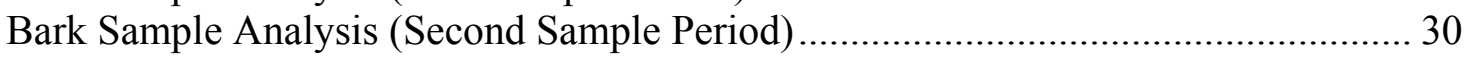

Pre-treatment vs. Post-treatment Analysis............................................................... 33

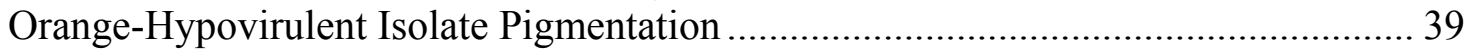

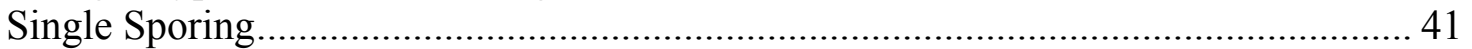

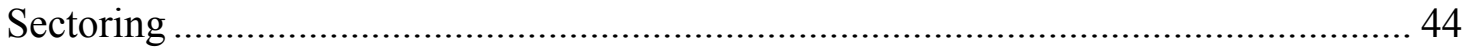

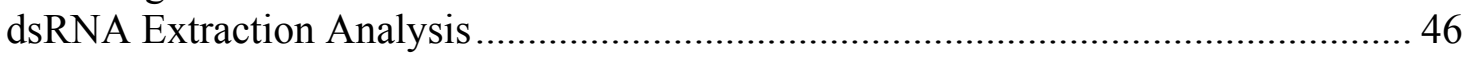

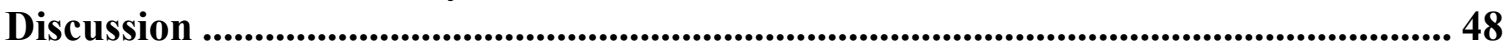

Summary ............................................................................................................................ 64

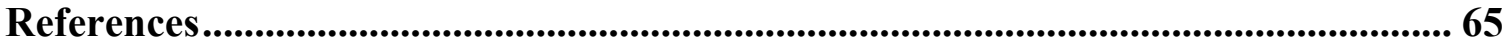

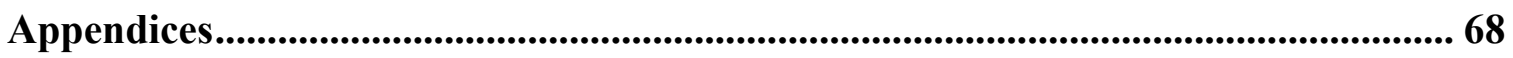




\section{List of Tables}

Tables $1-4$. Orange-virulent $(\mathrm{OV})$, orange-hypovirulent $(\mathrm{OH})$, and brown-hypovirulent (BH) conidia recovered from November 2002 and May 2003 sampling periods.

Percentages are indicated in parenthesis.

Table 5. Sectoring combinations isolated from the May 2003 sampling period. Each

treatment represents the number of sector types observed from 286 isolations 


\section{List of Figures}

Figure 1. An interior view of the MeadWestvaco research plot. .................................. 11

Figure 2. Covering material applied to half of the cankers in the study....................... 13

Figure 3. Scratching cankers with a box cutter and a metal template as a guide............. 14

Figure 4. Application of hypovirulent inoculum to punch wounds at the canker margin.

Figure 5. Morphologies of the virulent canker inciting isolate (MC-2), and the

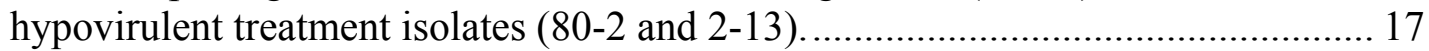

Figure 6. Bone marrow biopsy instrument used for removal of bark plugs. .................. 19

Figure 7. Sampling scheme used for the November 15, 2002 sampling period............. 21

Figure 8. Sampling scheme used for the May 28, 2003 sampling period....................... 22

Figure 9. Microtiter dish of bark samples with nickel-plated wire mesh screen attached.

Figure 10. Average canker expansion from time of treatment $\left(\mathrm{T}_{0}\right)$ to first sample period $\left(\mathrm{T}_{1}\right)$. 28

Figure 11. Average canker expansion from time of treatment $\left(\mathrm{T}_{0}\right)$ to second sample period $\left(\mathrm{T}_{2}\right)$...... 28

Figure 12. Percentage of orange-virulent $(\mathrm{OV})$, orange-hypovirulent $(\mathrm{OH})$, brownhypovirulent $(\mathrm{BH})$, and non-Cryphonectria $(\mathrm{C})$ isolates recovered from compatible treatments sampled on November 15, 2002.

Figure 13. Percentage of orange-virulent $(\mathrm{OV})$, orange-hypovirulent $(\mathrm{OH})$, brownhypovirulent $(\mathrm{BH})$, and non-Cryphonectria $(\mathrm{C})$ isolates recovered from incompatible treatments sampled on November 15, 2002. 30

Figure 14. Percentage of orange-virulent $(\mathrm{OV})$, orange-hypovirulent $(\mathrm{OH})$, brownhypovirulent $(\mathrm{BH})$, and non-Cryphonectria $(\mathrm{C})$ isolates recovered from compatible treatments sampled on May 28, 2003.

Figure 15. Percentage of virulent $(\mathrm{OV})$, orange-hypovirulent $(\mathrm{OH})$, brown-hypovirulent $(\mathrm{BH})$, and non-Cryphonectria $(\mathrm{C})$ isolates recovered from incompatible treatments sampled on May 28, 2003.

Figure 16. Percentage of orange-virulent $(\mathrm{OV})$, orange-hypovirulent $(\mathrm{OH})$, brownhypovirulent $(\mathrm{BH})$, and non-Cryphonectria $(\mathrm{C})$ isolates recovered from compatible treatments following the November 2002 sample. Panel A, positions 13-24 (pretreatment). Panel B, positions 1-12 (post-treatment).

Figure 17. Percentage of orange-virulent $(\mathrm{OV})$, orange-hypovirulent $(\mathrm{OH})$, brownhypovirulent $(\mathrm{BH})$, and non-Cryphonectria $(\mathrm{C})$ isolates recovered from incompatible treatments following the November 2002 sample. Panel A, positions 13-24 (pretreatment). Panel B, positions 1-12 (post-treatment). 36

Figure 18. Percentage of orange-virulent $(\mathrm{OV})$, orange-hypovirulent $(\mathrm{OH})$, brownhypovirulent $(\mathrm{BH})$, and non-Cryphonectria $(\mathrm{C})$ isolates recovered from compatible treatments following the May 2003 sample. Panel A, positions 19-24 (pretreatment). Panel B, positions 1-18 (post-treatment).

Figure 19. Percentage of orange-virulent $(\mathrm{OV})$, orange-hypovirulent $(\mathrm{OH})$, brownhypovirulent $(\mathrm{BH})$, and non-Cryphonectria $(\mathrm{C})$ isolates recovered from incompatible 
treatments following the May 2003 sample. Panel A, positions 19-24 (pretreatment). Panel B, positions 1-18 (post-treatment).

Figure 20. Morphologies of high (A) and low (B) pigmented orange-hypovirulent isolates.

Figure 21. Agarose gel analysis indicating presence of dsRNA in high and lowpigmented isolates. From left to right, Lanes 1 and 2 represent dsRNA-containing isolates 12-T-17 (high) and 9-B-11 (low) respectively. Lane 3 represents a dsRNAfree virulent isolate.

Figure 22. Results of apple pathogenicity test with a low pigmented (9-B-11), a highpigmented (12-T-17) and a virulent isolate.

Figure 23. Differences in number of colony sectors on GYE/A and PDA media. A. represents compatible treatments while B. represents incompatible treatments. Each treatment represents the number of sectors observed from 286 isolations.

Figure 24. Agarose gel analysis indicating presence or absence of dsRNA. From left to right, lanes 1, 2, 7 and 8 represent dsRNA-containing orange-hypovirulent isolates, lanes 4 and 5 represent dsRNA-containing brown-hypovirulent isolates and lanes 3 and 6 represent dsRNA-free orange-virulent isolates. 


\section{List of Appendices}

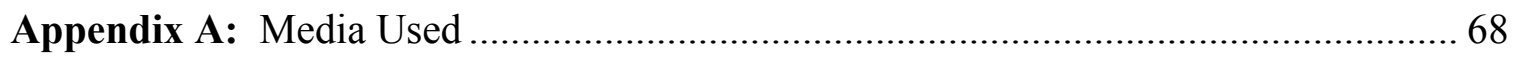

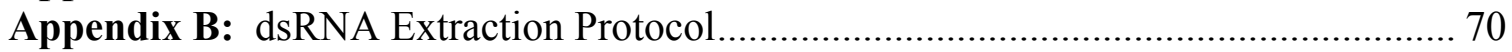

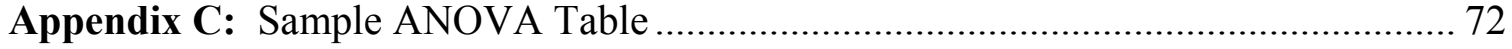


Introduction

The chestnut blight epidemic in North America is considered by many to be the most devastating North American ecological disaster in recorded history. The pathogen, Cryphonectria parasitica, was introduced into the United States on nursery stock from The Orient. Prior to its introduction, American chestnut (Castanea dentata) was a dominant tree species throughout the eastern hardwood forests. C. parasitica spread so rapidly that, by the mid-1950's, virtually all mature American chestnut within the species' natural range had succumbed to the disease. American chestnut now survives mainly as sprouts from old stumps and rooting systems and is presently unable to regain its former dominance in forest canopies.

Disease progress in Europe initially was similar to that in North America. However, there was a shift in the progression of the disease from stem-girdling to nonlethal, superficial cankers. This led to the discovery of the hypovirulence phenomenon when researchers first noted spontaneous healing of cankers and then observed that the morphology and pathogenicity of $C$. parasitica isolates were altered. Further work determined that hypovirulence was a cytoplasmic trait that could be transferred to other isolates via anastomosis. The phenomenon of hypovirulence was determined to be the result of cytoplasmically borne infectious dsRNA's that are transmissible and now are referred to as hypoviruses. Infection of the blight fungus by hypoviruses has been deemed responsible for the recovery and survival of chestnut in European orchards.

The situation in Europe resulted in an increasing interest in using hypoviruses as biocontrol agents for chestnut blight. However, one limitation to hypovirulence-based biological control appears to be a system of vegetative incompatibility that exists in 
natural populations of $C$. parasitica. This system restricts the fungus from transferring nuclei and cytoplasmic elements during vegetative growth, thereby restricting hypovirus transmission. In Europe, fewer vegetative compatability types exist in the $C$. parasitica fungal population; this feature may have permitted hypoviruses to be more successful at regulating the disease there.

Other factors undoubtedly have contributed to the failure of hypoviruses to establish as biocontrol agents in North America and their success in Europe. Some of these may include the reduction in sexual and asexual sporulation common to hypovirulent strains on both continents, a more resistant European host and different environmental conditions. The lack of a proper method to introduce hypovirulent strains into virulent $C$. parasitica cankers may be a further reason why attempts at biological control in North America largely have been unsuccessful. The primary objective of this study was to evaluate methods of introducing hypoviruses into virulent cankers on American chestnut trees using hypovirulent isolates that were either vegetatively compatible or incompatible with the treated canker. To meet this objective, twelve hypovirus introduction methods were tested. 


\section{Literature Review}

Historically, American chestnut, Castanea dentata (Marsh.) Borkh., was a major component of upland forests throughout the Appalachians until the inadvertent introduction of the chestnut blight fungus, Cryphonectria parasitica (Murr.) Barr, into North America. The chestnut blight fungus was unintentionally transported on oriental chestnut seedlings brought into New York for landscape use and orchard stock (Griffin, 1986). The fungus probably was introduced into North America before the turn of the century (Kuhlman, 1978), although it wasn't reported until 1904, when the fungus was first noticed in the Bronx Zoological Park (Merkel, 1905).

Murrill originally named the causal organism of chestnut blight, Diaporthe parasitica, in 1906. Six years later, Anderson and Anderson (1912) reclassified the causal organism into the genus Endothia. Barr (1978) changed the genus name when she placed the fungus into the genus Cryphonectria.

The origin of the chestnut blight fungus remained unknown until 1917 when observations indicated Asian chestnut seemed to have more natural resistance to the disease than did American chestnut (Shear et al., 1917), implicating an Asiatic origin for the pathogen (Kuhlman, 1978). Presumably, when host and pathogen evolve together, selection pressure allows for the development of resistance to the pathogen.

Prior to the introduction of the fungus, American chestnut dominated woodlands from Maine, south to Georgia and from the Piedmont west to the Ohio Valley. In surveys of the most concentrated areas, chestnut accounted for approximately $25 \%$ of the hardwood species (Braun, 1950). Not only was there an abundance of American chestnut trees in the forest, mature chestnuts averaged up to five feet in diameter and up to one- 
hundred feet tall. Many specimens of eight-to-ten feet in diameter were recorded, and there were rumors of trees even larger. Chestnut had a straight, dramatic grain and the wood was somewhat like oak in appearance though not as strong or dense; but its lightness, workability, and stability made it valuable for building, furniture, and woodworking. Its resistance to decay was especially valued in uses such as railroad ties and utility poles (Kuhlman, 1978). Chestnut was readily available and easy to work with. Split-rail fences, which are now common in showy restorations of frontier settlements, were made of chestnut. The tannins in its bark and wood added to the demand for chestnut to supply leather tanneries. Chestnuts themselves were an important food for humans, livestock and the animals that roamed the hardwood forests.

The demise of the American chestnut has been noted as one of the most significant ecological tragedies known. By the 1950s, virtually all-mature American chestnuts had succumbed to the disease. A contiguous natural range of chestnut, the lack of host genetic resistance and the capability of the fungus to live saprophytically are just a few of the contributing factors in the chestnut blight epidemic. American chestnut now exists mainly as sprouts from old stumps and root systems.

Wounding of the bark surface reportedly is essential for blight infection. Infections are initiated by either conidia or ascospores. Ascospores are disseminated by wind while insects, birds, and rain-splash disseminates conidia (Heald and Gardner, 1913; Scharf and DePalma, 1981; Russin et al., 1984). When spores germinate in bark wounds, hyphae spread by development of a mycelial fan under the bark. According to Griffin (1986), the initial lesion in American and Asian chestnut is surrounded by a water-impermeable wound periderm-induction barrier that limits the growth of individual 
hyphae, but not of the hyphae aggregates or mycelial fans. Mature wound periderm, however, stops the growth of mycelial fans. Wound periderm lies adjacent to the lignified zone and is formed earliest in the deepest portions of the wound, near the vascular cambium. It has a periclinal orientation in the inner bark areas and an anticlinal orientation in the outer bark area. Canker enlargement occurs because mycelial fans penetrate these areas of periclinal and anticlinal wound periderm before they are fully formed. This leads to canker development in the outer and inner bark regions, which is lethal to the trees when the canker girdles the stem and kills the vascular cambium.

In the 1950's, an Italian plant pathologist observed, "spontaneous healing" of chestnut blight cankers on European chestnut (C. sativa (Mill.)) (Biraghi, 1953). Biraghi noted that healing cankers appeared only in areas of early infection, approximately 15 years after the original onset of disease (Mittempergher, 1978). One of Biraghi's initial hypotheses to explain these healing cankers was the loss of pathogenicity in the fungus. These observations gained the attention of a French mycologist, Jean Grente, who described a variety of unusual strains of $C$. parasitica associated with the healing cankers on European chestnut (MacDonald and Fulbright, 1991). Grente observed that isolates of C. parasitica, taken from the abnormal cankers, had different morphological characteristics than the normal, virulent isolates of $C$. parasitica. Isolates from the abnormal cankers appeared lightly pigmented in culture as opposed to the normal, brightly orange-pigmented isolates of the virulent form of the fungus. These abnormal isolates also formed more limited stroma, and they seemed to be less virulent in pathogenicity studies than normal isolates (Grente and Berthelay-Sauret, 1978). Based upon these observations, Grente and Berthelay-Sauret (1978) coined the term 
"hypovirulent" to describe the abnormal isolates. Elliston (1982) further defines hypovirulence as "any state of disease producing capacity less than the norm".

Hillman et al. (1995) officially defined the infectious agents. Taxonomically, hypoviruses were classified into the family Hypoviridae and the genus Hypovirus. All confirmed members of the genus Hypovirus infect $C$. parasitica and cause reductions in fungal virulence as well as altered fungal morphology. Morphologically, hypovirulent isolates possess pleomorphic membrane-bounded vesicles within cytoplasm that are 50$80 \mathrm{~nm}$ in diameter, and contain dsRNA; the replicative component of many fungal viruses. They are the only virus particles that have successfully been extracted from infected fungal tissue.

The ability of hypovirulent isolates to convert "normal" virulent isolates to hypovirulent isolates lead to potential uses of these abnormal isolates in biological control of the chestnut blight fungus. Mittempergher (1978) reported on the status of chestnut blight in Italy and noted that hypovirulent isolates of $C$. parasitica resulted in significant control of the blight in Italy and France and that chestnut blight is no longer considered a serious threat to $C$. sativa in these countries.

Hypovirulent isolates also have been isolated from surviving chestnut trees and stands of chestnut in Michigan (Fulbright et al., 1983). In a few of these surviving stands, the trees are recovering from chestnut blight and almost all signs of C. parasitica have disappeared. This may be due to the fact that these stands are located in central and western Michigan, outside the natural range of $C$. dentata, which reached only into southeastern Michigan (MacDonald and Fulbright, 1991). Hypovirulent strains of $C$. 
parasitica have been isolated from several sites in Michigan, as well as from Maryland, New York, Tennessee, Virginia and West Virginia (Jaynes and Elliston, 1982).

Hypoviruses have been more effective in controlling C. parasitica in Europe than in North America. Many factors may be associated with this differing response. One factor is the complexity of the fungal population. A system of vegetative incompatibility exists in C. parasitica that restricts the transfer of nuclei and cytoplasmic elements during vegetative growth, and likewise prevents the successful transmission of hypoviruses (Anagnostakis, 1977). Hyphal fusion (anastomosis) is necessary for hypoviruses to be transmitted successfully. In culture, a clearly delineated barrier or barrage occurs when incompatible isolates are paired closely on an agar medium. When transmission of hypoviruses fails to occur, hypovirus establishment is difficult. The Appalachian region has a large number of vegetative incompatibility groups. The efficacy of hypovirus dissemination is inversely proportional to vegetative incompatibility diversity. Vegetative incompatibility has been implicated as one reason biological control has met with limited success in the eastern United States.

MacDonald and Double (1978) examined the complexity of C. parasitica in West Virginia. They examined 202 C. parasitica isolates and found that $89 \%$ of the isolates could be classified into 14 different vegetative-compatibility (VC) groups. MacDonald et al. (1982) reported 37 VC groups present in other areas of West Virginia. Liu and Milgroom (1996) conducted a field study in Maryland and discovered 31 different VC groups from a total of 58 isolates. Other studies have been conducted on vegetative-compatibility and a total of more than $80 \mathrm{VC}$ groups have been identified as compared to $9 \mathrm{VC}$ groups identified in Italy and the $20 \mathrm{VC}$ groups identified in France; 
areas where recovery from chestnut blight is occurring (Anagnostakis and Waggoner, 1981).

Another factor that may influence the successful introduction and establishment of hypoviruses into the North American population of $C$. parasitica is their method of introduction into virulent $C$. parasitica cankers. The canker margin-punch-method, first used by Grente and Berthelay-Sauret (1978), has been effective in introducing hypoviruses in many field studies, yet this technique is time consuming and labor intensive. The margin-punch-method requires introducing the hypoviruses at different intervals (spacings) around the canker margin. Jaynes and Elliston (1980) compared four methods of hypovirus introduction; all were variations of the punch method. They compared a conidial spray on wounded and non-wounded stems, an agar slurry of eight hypovirulent strains applied to four holes around the canker margin, eight holes punched into canker margin with a different hypovirulent strain applied to each hole, and agar plugs of one of eight strains inserted into four holes around the canker margin. By measuring the increase in canker size during the study, they concluded that two of the methods, the slurry treatment and all eight strains applied around the canker margin, were most effective. Each of these methods, which involved punching the canker margin, had a significantly lower average canker area than the other two methods, however the two most effective treatments could not be statistically separated from each other. They also concluded that wounding the cankers prior to treatment was more effective in introducing hypoviruses than without wounding.

Hobbins (1985) and Balbalian (1998) introduced hypoviruses into virulent cankers via colonized hypovirulent chestnut bark patches. Hobbins' objective was to 
provide a persistant source of hypovirulent inoculum with the use of bark patches. To accomplish the objective, sterilized bark patches were inoculated in the laboratory with hypovirulent fungal strains. After colonization, the bark patches were mounted $10 \mathrm{~cm}$. above the cankers on study trees. He discovered that this treatment method was useful in delivering hypovirulent inoculum, especially when the bark patch inoculum source was vegetatively compatible with cankers. This method was intended to simulate natural sources of inoculum without wounding, however the bark patches rely heavily on biotic and abiotic factors to disseminate inoculum. Balbalian (1998) also treated cankers with hypovirulent bark patches. Although the nature of her study was to measure hypovirus transmission when vic gene differences between inoculum and cankers were known, using the bark patches as a means of hypovirus delivery resulted in less hypovirus transmission than achieved by Hobbins.

Jaynes and DePalma (1982) treated natural infections with mixtures of hypovirulent isolates by using the canker punch method developed by Grente and by spraying conidia from a backpack mist blower. The authors concluded that inoculating pre-existing cankers with mixtures of hypovirulent isolates is a viable method of treating cankers and can overcome some of the compatibility barriers. They also concluded that more appropriate mixtures of hypovirulent treatment strains and more efficient application techniques are required for practical control of cankers on treated stems.

Other procedures to adequately introduce hypoviruses into virulent $C$. parasitica cankers have not been tested and warrant investigation and comparison to the canker margin punch method. Other methods may be easier, less time consuming and more effective than those previously used. The objective of this research, therefore, was to 
evaluate a variety of hypovirus introduction procedures. Canker treatment inoculum that was vegetatively compatible and incompatible with the canker inciting isolate was used in this study 
Materials and Methods

\section{Site Description}

Healthy American chestnut sprouts (Castanea dentata) located in compartment 18 of the MeadWestvaco Timberlands were selected for this study. Trees were located in a stand adjacent to Beaver Creek tract \# 819, approximately 7 miles from Beverly, West Virginia. The site was cut over in 1996, and was comprised mainly of American chestnut and black cherry sprouts (Prunus serotina). There were a small number of other competing hardwoods on this site (Figure 1).

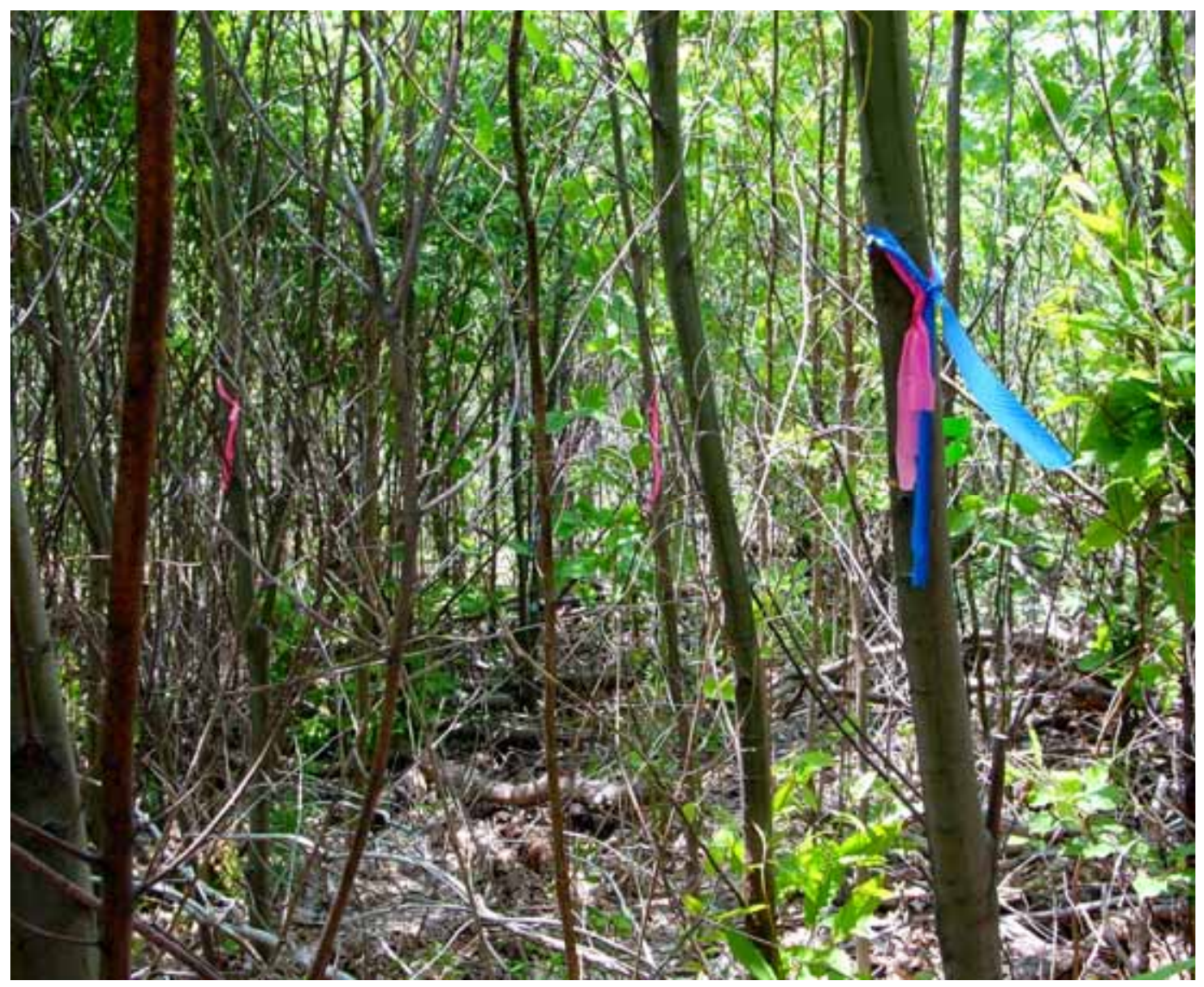

Figure 1. An interior view of the MeadWestvaco research plot. 


\section{Study Design}

Seventy-two American chestnut trees, ranging in size from 4.5 to 8.5 in. DBH were used in this study. Artificially established cankers were initiated on all trees on June 11, 2002 with an orange-pigmented, virulent isolate (MC-2) of Cryphonectria parasitica. Inoculum of MC-2 was grown on Potato Dextrose Agar (PDA) (Appendix A) 6-8 days prior to inoculation. Cankers, established at 2.5 and 5 feet above the ground, were initiated by removing an 11-mm diameter bark disk from the stem and inserting a similar size piece of 7-day-old mycelium of the canker inciting strain, MC-2. All inoculation sites were covered with tape to prevent desiccation. On August 23, 2002, 11 weeks after initiation, cankers were challenged with either a brown-pigmented compatible (80-2) or incompatible (2-13) hypovirulent $C$. parasitica isolate.

Three direct hypovirulent introduction treatments, one non-invasive and two invasive, were employed. The non-invasive treatment consisted of a paintbrush application of an agar slurry containing the appropriate hypovirulent isolate. Cankers were painted evenly with the hypovirulent inoculum slurry. One-half of the cankers treated by this method were covered with an absorbent underpad $(15 \mathrm{~cm} \times 12.5 \mathrm{~cm})$ (Fisherbrand Scientific ${ }^{\circledR}$ ) (Figure 2). The other half of the cankers remained uncovered. In an effort to reduce tree-to-tree inoculum interactions, trees treated with compatible hypovirulent inoculum were physically separated, approximately 20 meters, from those treated with incompatible hypovirulent inoculum.

One invasive treatment consisted of wounding the canker with a box cutter, followed by application of the hypovirus containing strain with a paintbrush (Figure 3). A metal template was used as a guide to produce a consistent number of vertical and 
horizontal lines. Following wounding, the hypovirulent inoculum slurry was distributed evenly with a paintbrush on the canker faces and either covered or uncovered.

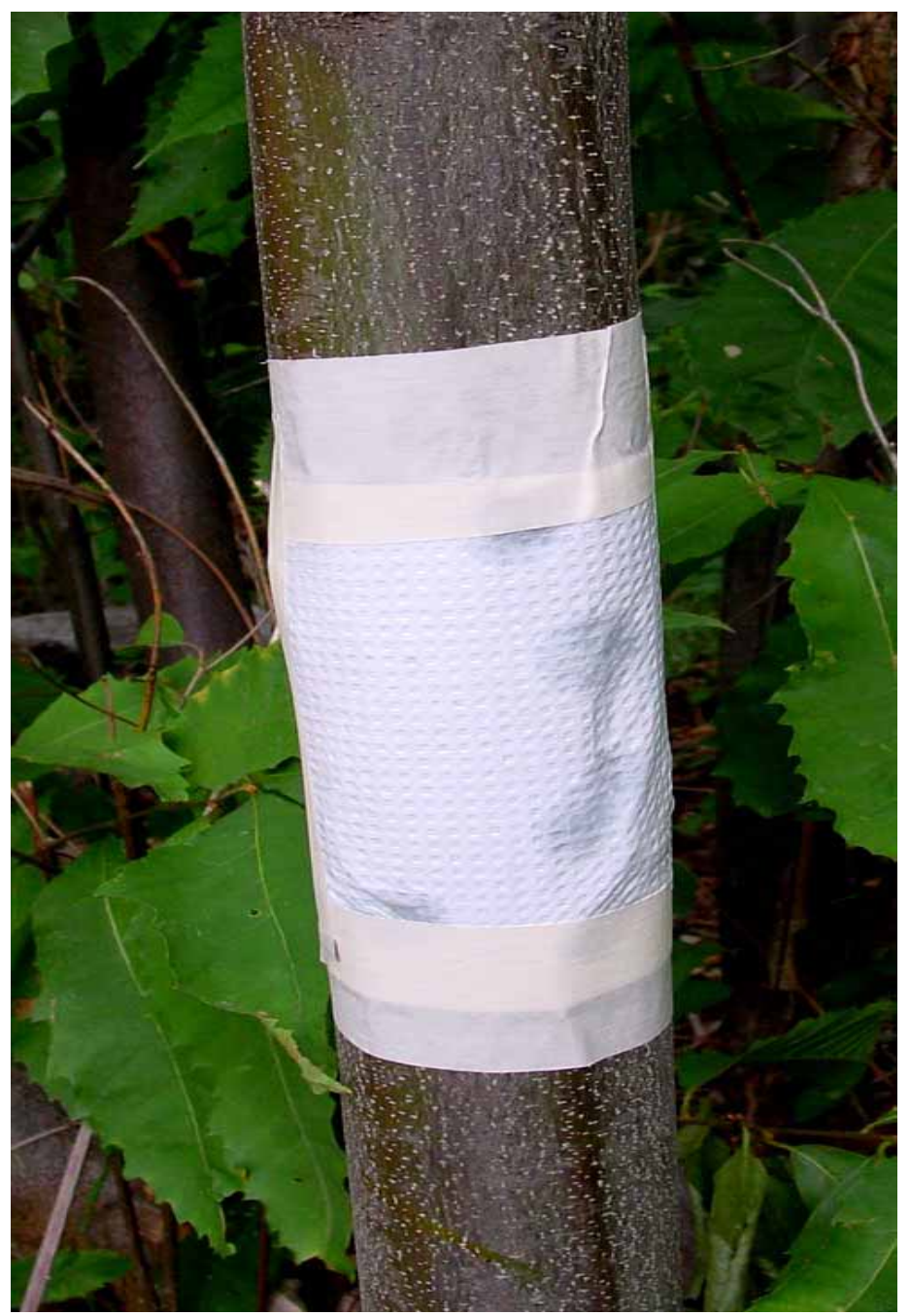

Figure 2. Covering material applied to half of the cankers in the study.

The second invasive treatment utilized the margin-punch method. This treatment procedure was applied using the method described by Grente and Berthelay-Sauret (1978). When challenging a canker by this method, a series of punch wounds were made around the canker perimeter with a hammer and an 11-mm leather punch. Hypovirulent agar slurry was applied to the margin of these cankers by dispensing inoculum from 500- 
$\mathrm{ml}$ plastic squirt bottles (Figure 4). Slurry-filled wounds were either covered or uncovered.

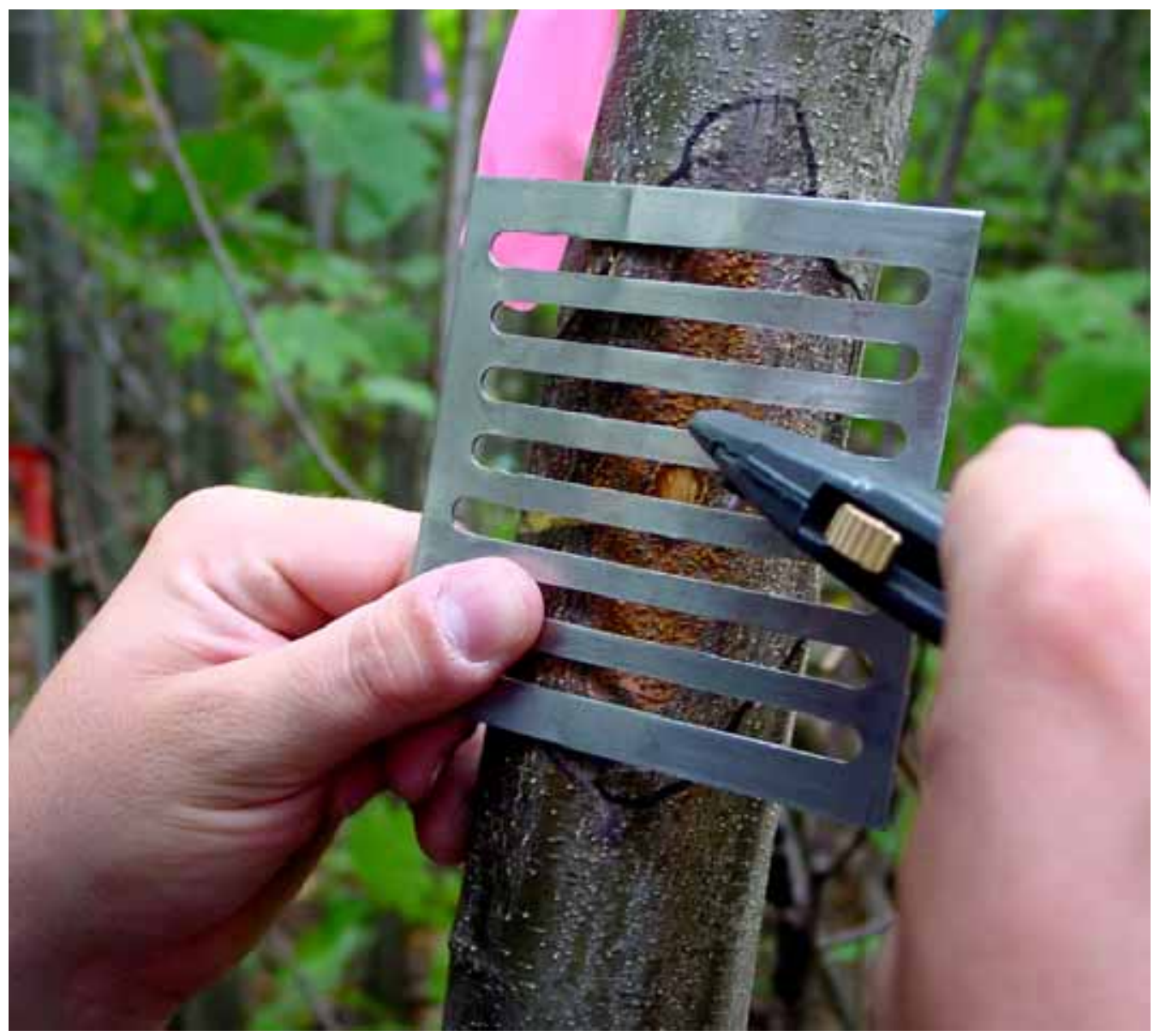

Figure 3. Scratching cankers with a box cutter and a metal template as a guide.

For all treatments, the same treatment procedure was applied to both cankers on a tree. The canker covering material: (1) protected cankers from rain and other biotic or abiotic factors; and, (2) was used to determine if canker coverings improved survival and subsequent transmission of hypovirus. The covering was removed 18 days after treatment. A preliminary test showed that the absorbent underpads enabled C. parasitica to survive and stay moist better than other coverings tested (i.e. wax paper, a Supplex ${ }^{\circledR}$ 
material, brown paper bags, vinyl flagging, freezer paper, aluminum foil, heavy-duty plastic and lightweight plastic bags).

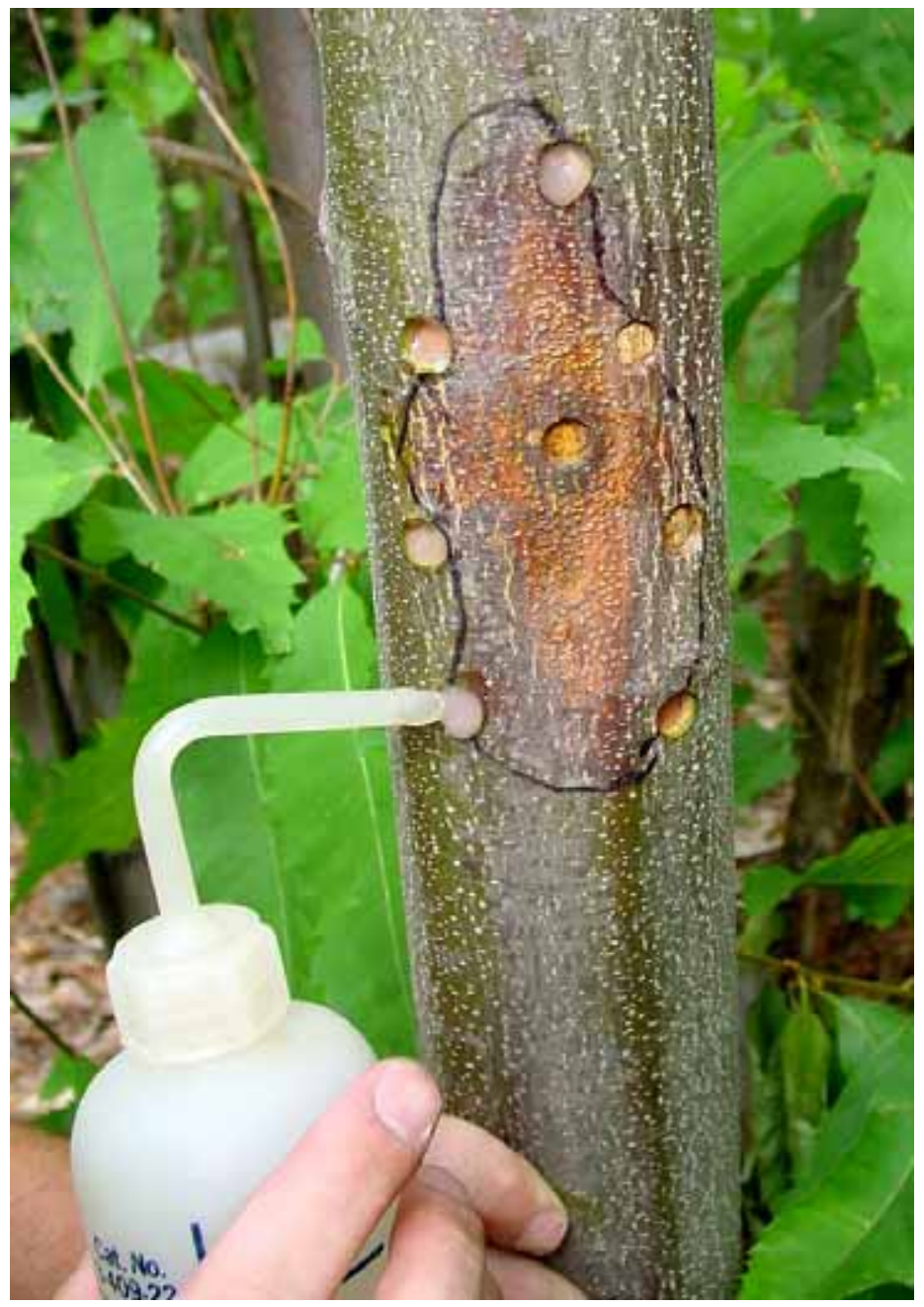

Figure 4. Application of hypovirulent inoculum to punch wounds at the canker margin.

\section{Isolate Selection}

Cankers were initiated with an orange-pigmented virulent strain of C. parasitica (MC-2) that was isolated from a canker in a chestnut planting in Marion County, West Virginia. Cankers were challenged with either a vegetatively compatible or incompatible 
brown-pigmented isolate containing a CHV-1 hypovirus (Hillman, et al., 1995). The compatible hypovirulent isolate, 80-2, was isolated in 1982 from a field study conducted in Pocahontas County, West Virginia. This isolate is pigmented brown and arose by an in vivo infection with a European hypovirus, $(\mathrm{CHV}-1)$. The incompatible hypovirulent isolate, 2-13, was created in vitro on cellophane (Flexal Corporation) by pairing a brown virulent isolate and an orange hypovirulent isolate (CHV-1) (9-A-1-1). This pairing resulted in a brown-pigmented hypovirulent isolate that was incompatible with the canker inciting MC-2 strain. The brown virulent background isolate, (West Virginia vegetative compatibility group F), was generated as part of a 1978 perithecial production study and arose from a single ascospore. The orange hypovirulent isolate (9-A-1-1) was discovered in a West Virginia field study conducted in 1982. The hypovirus associated with this isolate was transmitted to the brown-pigmented virulent isolate by pairing on cellophane creating the incompatible brown hypovirulent isolate. The unique morphologies of $\mathrm{MC}$ 2, 80-2 and 2-13 are shown in Figure 5. Preliminary in vitro pairing experiments confirmed that isolate MC-2 was readily converted by the compatible hypovirulent isolate $80-2$ but not by the incompatible hypovirulent isolate 2-13. 


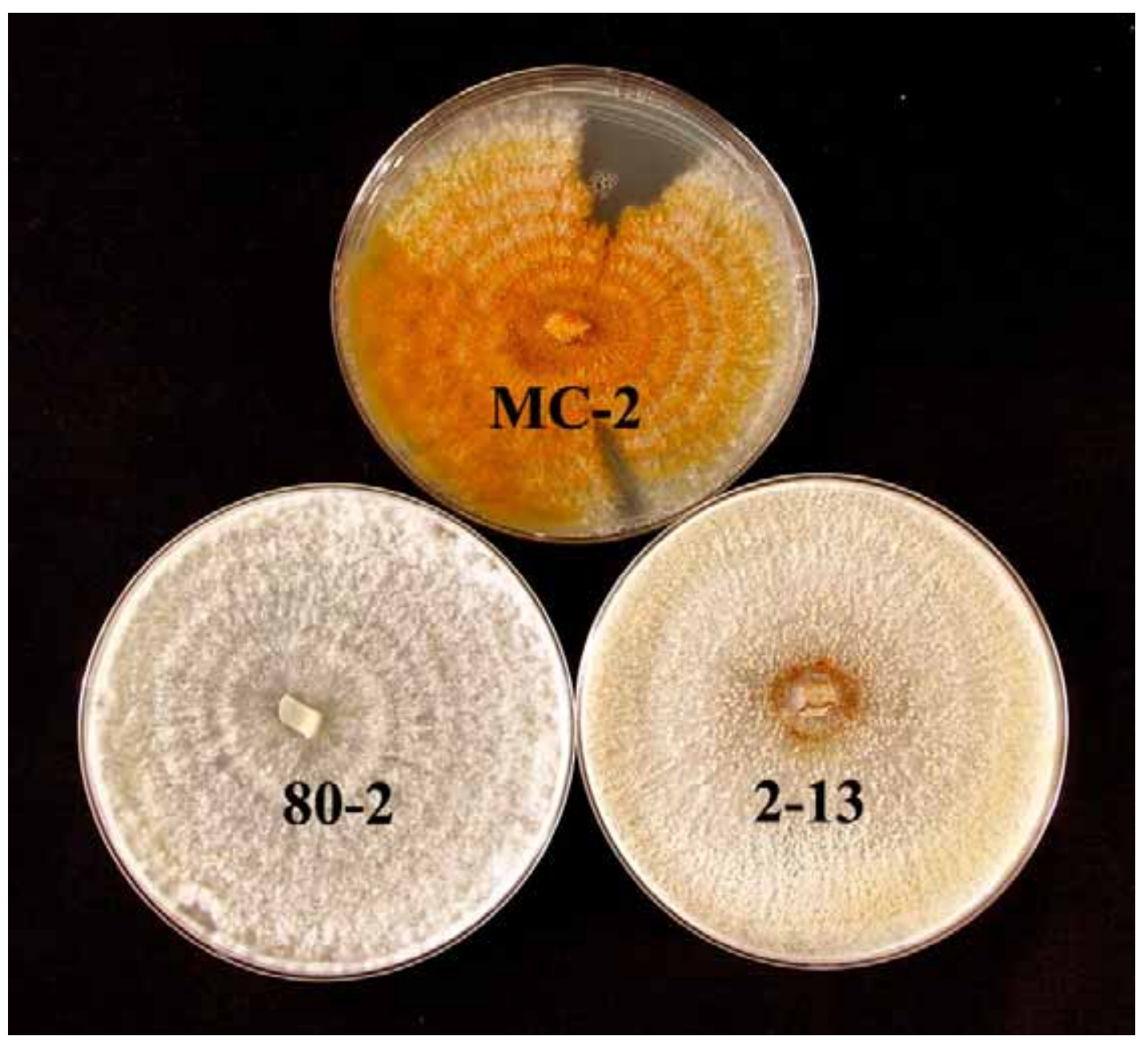

Figure 5. Morphologies of the virulent canker inciting isolate (MC-2), and the hypovirulent treatment isolates (80-2 and 2-13).

\section{Inoculum Preparation}

The canker inciting orange-virulent strain (MC-2) was grown on PDA in a $20^{\circ} \mathrm{C}$ incubator with a 16:8 hour photoperiod. Petri plates containing the virulent isolate were prepared 6 days prior to the field inoculations.

A liquid glucose yeast extract (GYE) medium (Appendix A) was used to prepare the hypovirulent treatment inoculum. Six hundred $\mathrm{ml}$ of liquid GYE was added to four wide-mouth 2-L Erlenmeyer flasks. Flasks were sealed with a double layer of aluminum 
foil and inverted $600-\mathrm{ml}$ beakers. Flasks were autoclaved for 24 minutes at $120^{\circ} \mathrm{C}$. After autoclaving, flasks were allowed to cool thoroughly and then inoculated. Two flasks were inoculated with mycelial plugs of the compatible hypovirulent strain (80-2) and two with the incompatible hypovirulent strain (2-13). The flasks were incubated at room temperature under 40-watt Sylvania ${ }^{\circledR}$ "Cool White" fluorescent lamps for 10 days. Following incubation, mycelium was harvested in an 8 in. Buchner funnel lined with a 40 $\mathrm{cm} \times 40 \mathrm{~cm}$ piece of cheesecloth. Excess liquid was removed via a vacuum pump attached to a 4-L Erlenmeyer flask. Mycelium was then added to a Waring ${ }^{\circledR}$ blender and combined with $450 \mathrm{ml}$ of a sterile $0.1 \%$ peptone solution (Appendix A). After blending, the peptone-mycelium solution was thickened to the consistency of applesauce by the addition of approximately $500 \mathrm{ml}$ of solidified $2.5 \%$ water agar and additional blending (Appendix A). The hypovirulent slurry mixtures were distributed to either 2-L plastic containers or to $500-\mathrm{ml}$ squirt bottles for field application. Slurries were refrigerated and used within two days of preparation.

\section{Canker Measurement and Sampling}

Canker margins were outlined with a black Sharpie ${ }^{\circledR}$ marker and measured (length and width) at the time of treatment (August 23, 2002), at the time of the $1^{\text {st }}$ sampling period (November 15, 2002), and the $2^{\text {nd }}$ sampling period (May 28, 2003). For statistical analysis, the length and width $(\mathrm{cm})$ of each canker was summed and divided by $2((\mathrm{~L}+\mathrm{W}) / 2)$ and the means of all replications were used to determine if hypovirus treatment had an effect on canker expansion. 
On November 15, 2002, twenty-four bark plugs were removed from each canker with a bone marrow biopsy instrument (Lee-Lok Instrument Co.) (Figure 6) for culture. Four samples were removed from the area directly adjacent to the canker initiation points, in the four cardinal directions (Figure 7). Eight plugs were removed from the area directly inside of the margin at the time of treatment. The final twelve plugs were removed from the area that grew beyond the treatment margin. Pre - treatment (positions $13-24$ ) and post - treatments (positions $1-12$ ) bark plugs also were analyzed to determine if hypovirus acquisition occurred within cankers. Bark plugs were removed and placed in a 96-well microtiter dish (Fisherbrand Scientific) so that the position of the sample in the canker could be recorded. The wells were then covered with tape and the bark samples were stored at $-20^{\circ} \mathrm{C}$ until analysis.

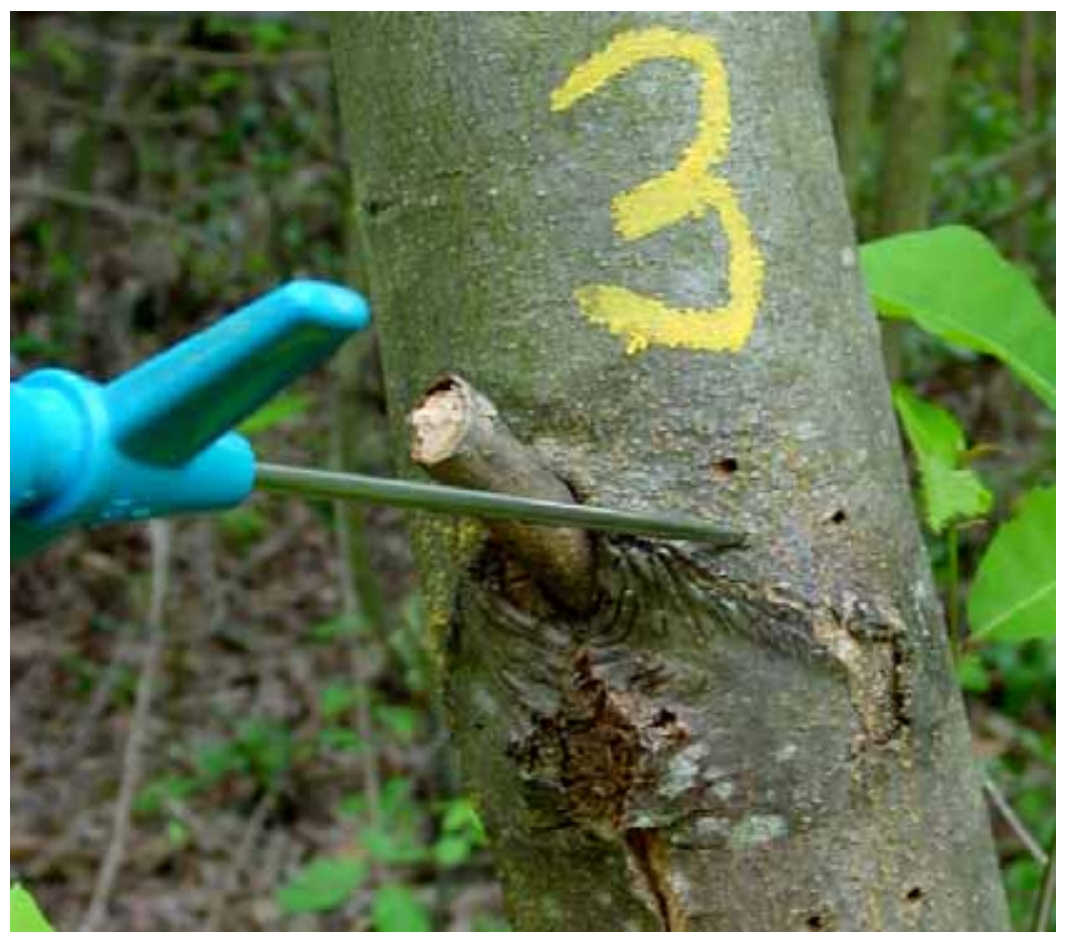

Figure 6. Bone marrow biopsy instrument used for removal of bark plugs. 
Two 11-mm bark disks were removed from each canker for evaluation of asexual spore production. One of the bark disks was removed from the area of the canker that grew prior to treatment and the other bark disk was removed from the area of canker expansion following treatment. Each of these disks was placed in a 24-well tissue culture plate and its position recorded. The wells were covered with tape and the bark disks were stored at $-20^{\circ} \mathrm{C}$ until analysis.

The second set of samples was taken on May 28, 2003. Twenty-four bark plugs were removed from each canker with the bone marrow biopsy instrument during this sampling; a different sampling scheme was employed (Figure 8). During this sampling, the outer twelve samples were taken from the leading edge of the canker. If canker expansion had ceased, the leading canker margin in May 2003 may not have been different from the November 2002 margin. Notes were made on whether the canker had expanded more than $1 \mathrm{~cm}$. Two samples were removed from the canker adjacent to the initiation point in the north-south direction. Four samples were removed from the area directly inside the perimeter of the cankers at the time of treatment. The final six samples were removed from the area of the canker that had developed between the time of treatment and the $1^{\text {st }}$ sampling period. Pre - treatment (positions 19-24) and post treatments (positions $1-18$ ) bark plugs also were analyzed to determine where hypovirus acquisition occurred within cankers.

Two 11-mm bark disks also were removed from each canker in the $2^{\text {nd }}$ sampling period. One bark disk each was removed from the area that grew prior to treatment and after treatment. Both the bark plugs and the bark disks were handled and stored at $-20^{\circ} \mathrm{C}$ as in the $1^{\text {st }}$ sampling period. 


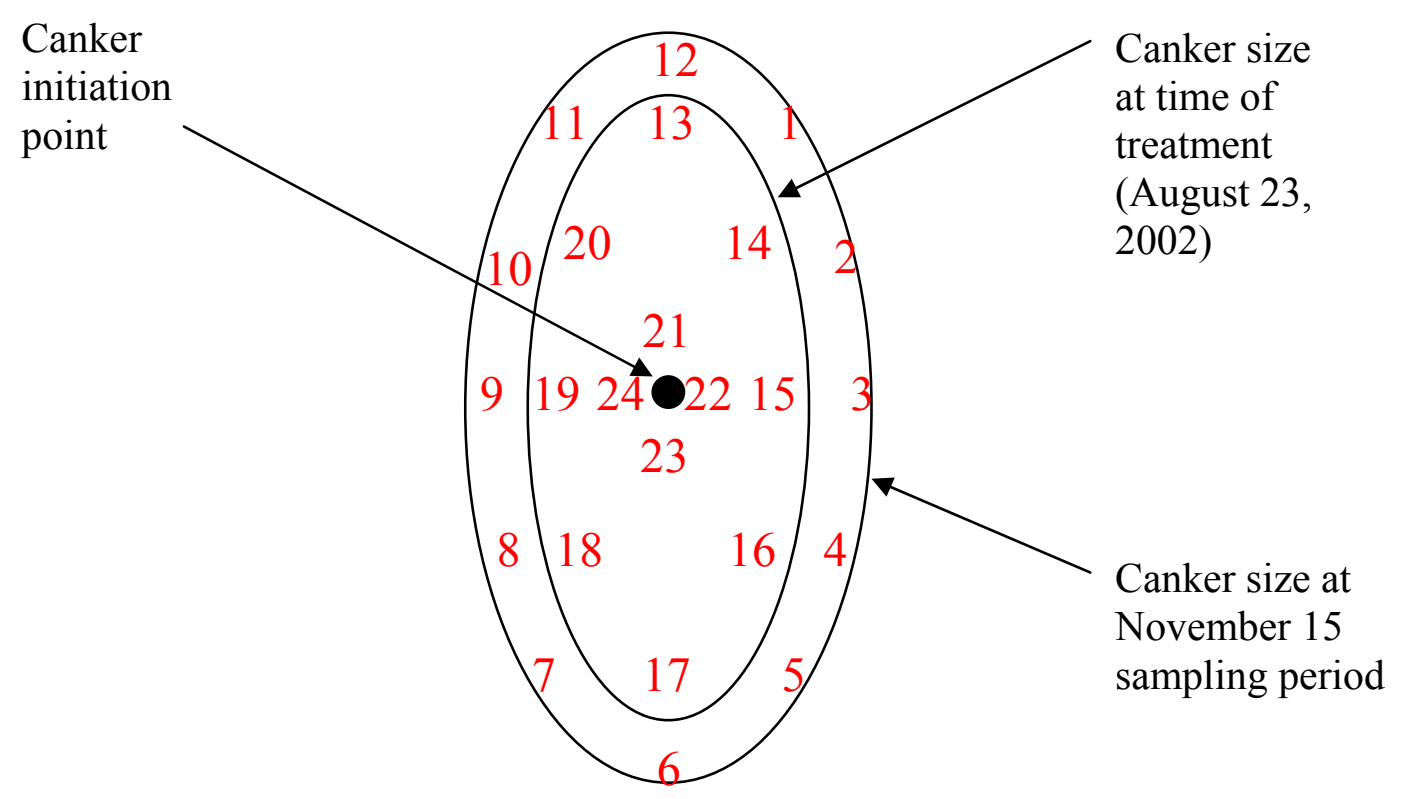

Figure 7. Sampling scheme used for the November 15, 2002 sampling period.

\section{Sample Processing}

Individual microtiter dishes were removed from the freezer and the bark plugs warmed to room temperature. Tape was removed and a nickel-plated wire mesh screen $(12 \mathrm{~cm} \times 7.5 \mathrm{~cm})$ (Fisherbrand Scientific) attached to the dishes with rubber bands (Figure 9). The plates were then submerged in a $0.6 \%$ sodium hypochlorite solution for 14 minutes. Bark plugs were then transferred (4 / plate) to glucose yeast extract agar amended with antibiotics (GYE/A) (Appendix A). Bark plugs were incubated at room temperature for 3-to-5 days. The resulting C. parasitica colonies were then transferred to PDA and grown at $20^{\circ} \mathrm{C}(16: 8$ photoperiod) for 7 - 10 days. 


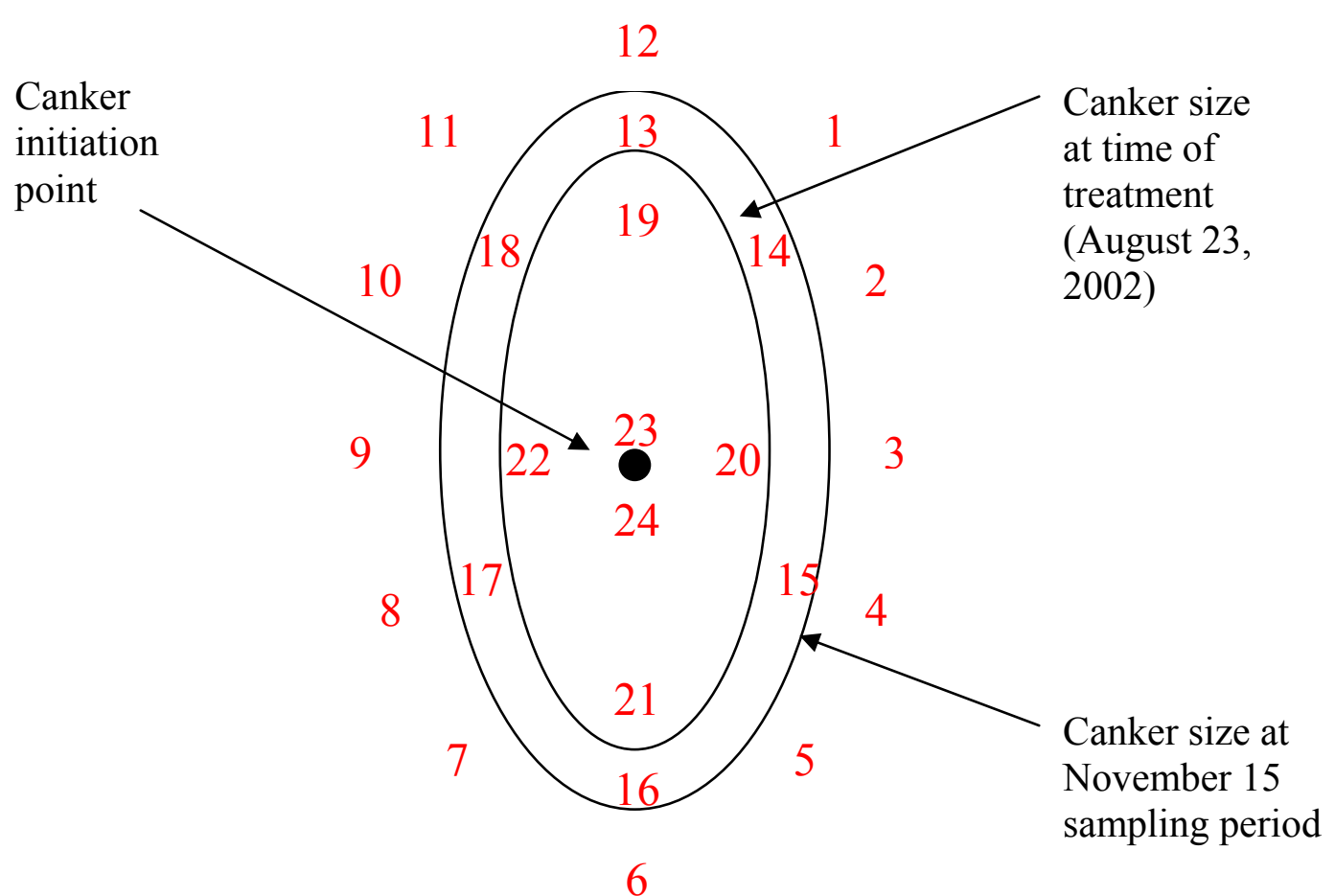

Figure 8. Sampling scheme used for the May 28, 2003 sampling period.

Isolate pigmentation and morphology of the fungal colonies from bark plugs were documented following incubation and used to determine whether hypovirus transmission had occurred. Successful transmission was indicated when lightly pigmented orange isolates were recovered, the result of anastomosis between the orange-virulent strain and a brown pigmented hypovirulent strain. Throughout the course of this study, orangehypovirulent converted isolates yielded differing levels of orange pigmentations. These isolates were classified as either low-pigmented or high-pigmented orange-hypovirulent isolates and were tested for presence of dsRNA and differences in pathogenicity. Further classification into low and high-pigmented isolates was determined after the parent isolates were single spored. When no hypovirus transmission occurred, either brownpigmented hypovirulent isolates were recovered (representing the treatment inoculum), or 
orange-virulent isolates were recovered, indicating either wild-type isolates or the cankerinitiation isolate.

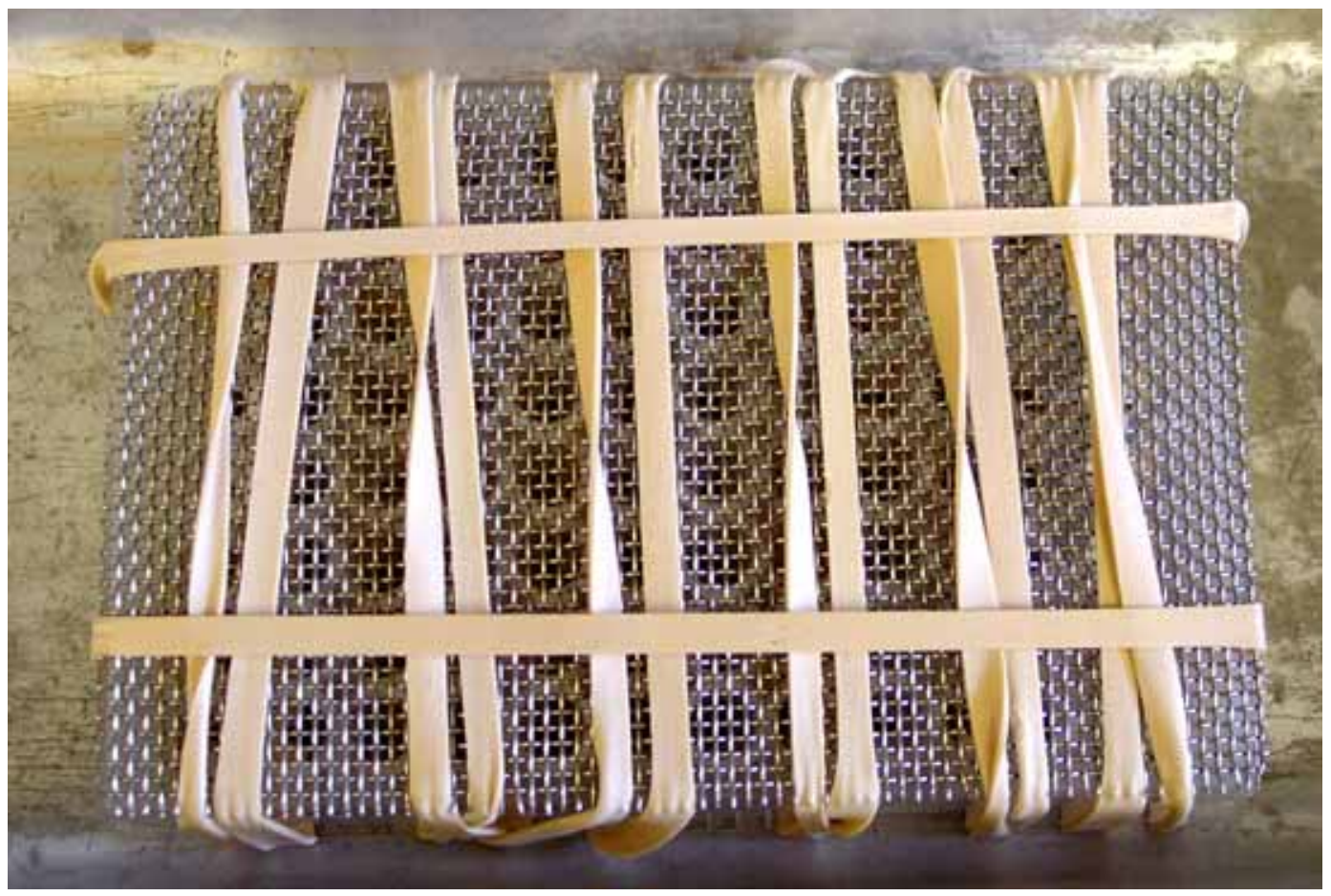

Figure 9. Microtiter dish of bark samples with nickel-plated wire mesh screen attached.

Sectored isolates also were noted during this study. These colonies consisted of sectors representing different morphology types including combinations of; OV (orangevirulent), $\mathrm{OH}$ (orange-hypovirulent) and $\mathrm{BH}$ (brown-hypovirulent). The primary reason for collecting data on the sectored isolates was to determine the frequency of bark plugs that contained different isolates on GYE/A and PDA and whether sectoring was more commonly associated with a particular treatment. The number of sectors in a bark plug colony on GYE/A was recorded approximately 7 days after subculturing to PDA. After incubation, the morphologies of sectored isolates that developed on PDA were recorded. 
Since sectoring was noticed after the onset of this study, sector data was gathered only on bark plugs from the May 2003 sampling period.

To determine if orange-virulent isolates, recovered from canker reisolations, represented the canker-inciting strain MC-2 or a wild-type isolate, recovered orange virulent isolates were paired with MC-2 on bromcresol green media (Powell, 1995) (Appendix A). This medium was selected because of the ease in ascertaining vegetativecompatibility. Four orange-virulent isolates were selected from each canker at random for vegetative compatibility typing. This test was conducted in darkness at room temperature for $7-10$ days.

\section{dsRNA Extractions}

Representative $\mathrm{OV}, \mathrm{OH}$ and $\mathrm{BH}$ isolates from each sample period were assayed for the presence of double-stranded ribonucleic acid (dsRNA). The purpose of the dsRNA extractions in this study was to confirm that the visual morphological categorization of colonies $(\mathrm{OV}, \mathrm{OH}$ or $\mathrm{BH})$ corresponded to the presence or absence of dsRNA. Mycelia for extraction were grown on cellophane (Flexal Corporation) overlayed on PDA at $20^{\circ} \mathrm{C}$ for 7 - 10 days (16:8 hour photoperiod). Mycelia were harvested and ground to powder in a cold mortar submerged in liquid nitrogen and stored in scintillation vials at $-20^{\circ} \mathrm{C}$ until analyzed. Nucleic acids were extracted using a modified version of the methods of Morris and Dodds (1979)(Appendix B). The dsRNA was analyzed by gel electrophoresis in $1.0 \%$ agarose stained with ethidium bromide. Gels were electrophoresed for 2 hours at $100 \mathrm{mV}$, examined under UV light (250nm) for presence or absence of bands, and photographed using Genesnap software (Syngene ${ }^{\circledR}$ ). 


\section{Single Sporing}

Single conidia were isolated from bark disks removed during both sampling periods, to assess virulent and hypovirulent conidial production. Asexual stroma were removed with an inoculation needle and placed in a watch glass containing $2 \mathrm{ml}$ of a $0.1 \%$ peptone solution. The stroma were then squashed with the inoculation needle and the $2 \mathrm{ml}$ peptone solution, containing conidia, was pipeted into a 2-ml microcentrifuge tube. The spore solution was serially diluted on plates of GYE/A; conidia were incubated at room temperature for two days. Fifty germinating conidia then were transferred to PDA and incubated for $7-10$ days at $20^{\circ} \mathrm{C}$ (16:8 hour photoperiod). After the incubation period, each resulting single spore colony was classified by pigmentation and morphology.

\section{Statistical Analysis}

A split plot design was used for data analysis in this study. ANOVA was used to analyze differences in recovery of orange-virulent, orange-hypovirulent and brownhypovirulent isolates. In these analyses, comparisons were made among canker wounding types (no wounding vs. wounding), canker coverings and isolate compatibility. Also, canker expansion data were analyzed. Within each comparison, a tree represented an individual experimental unit. When comparing pre-treatment vs. post-treatment isolate recovery, trees were divided into two sub plots. Differences in isolate recovery from the fall and spring sampling periods also were analyzed by separating trees into another sub plot. Orthogonal contrasts were used to further determine where significant 
differences occurred. All statistics were analyzed using the SAS statistical software package and compared at the $\alpha=0.05$ significance level. Statistical analysis was conducted with assistance from Dr. George Seidel, West Virginia University Agricultural Experiment Station statistician. A sample ANOVA table for $\mathrm{OH}$ isolate recovery is available in Appendix C. 


\section{Results}

One hundred forty-three of the 144 cankers established on June 11, 2002 grew. The one exception was due to an oversight and failure to initiate the canker. Cankers expanded at a uniform rate; by the time the treatments were applied, all cankers were approximately $8.7 \mathrm{~cm}((\mathrm{~L}+\mathrm{W}) / 2)$. All trees remained alive for the duration of the study, however, the number of natural infections markedly increased over the two growing seasons decreasing the vigor of some of the test trees.

\section{Canker Measurements}

When all treatments from $\mathrm{T}_{0}$ to $\mathrm{T}_{1}$ were compared, cankers treated with incompatible hypovirulent inoculum grew significantly more than cankers treated with compatible hypovirulent inoculum $(\mathrm{P}>\mathrm{F}=<0.0001)$ (Figure 10). However, wounded cankers expanded significantly less than non-wounded cankers among the compatible treatments $(\mathrm{P}>\mathrm{F}=0.0218)$.

When the same comparisons of canker growth were made from $T_{0}$ to $T_{2}$, similar results were obtained; cankers treated with incompatible inoculum grew significantly more than those treated with compatible inoculum, regardless of treatment $(\mathrm{P}>\mathrm{F}=$ 0.0015). There was significantly less expansion in wounded cankers than non- wounded cankers from $\mathrm{T}_{0}$ to $\mathrm{T}_{2}(\mathrm{P}>\mathrm{F}=<0.0001)$. All cankers treated with incompatible inoculum expanded similarly from $\mathrm{T}_{0}$ to $\mathrm{T}_{2}$. 


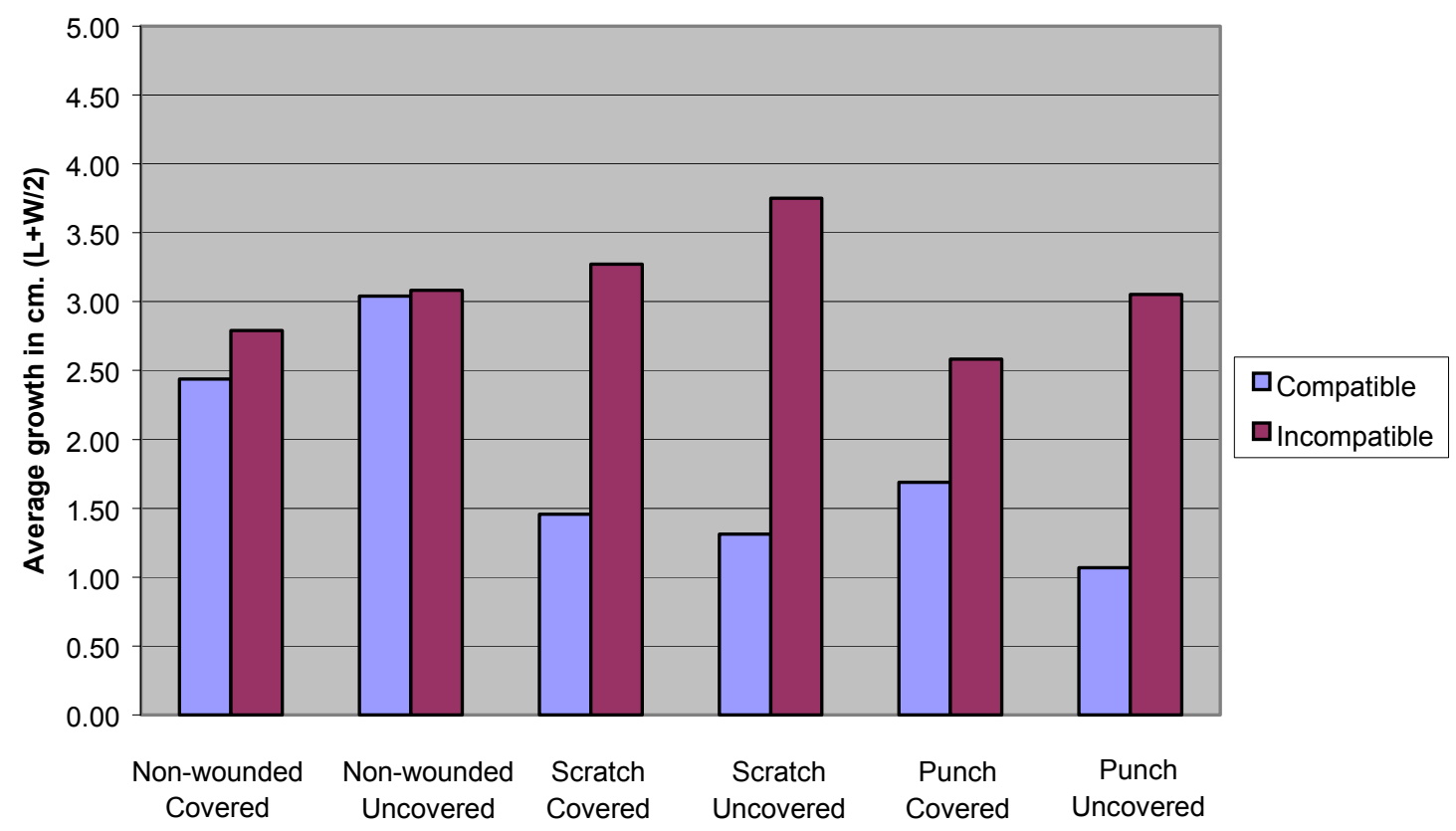

Figure 10. Average canker expansion from time of treatment $\left(\mathrm{T}_{0}\right)$ to first sample period $\left(\mathrm{T}_{1}\right)$.

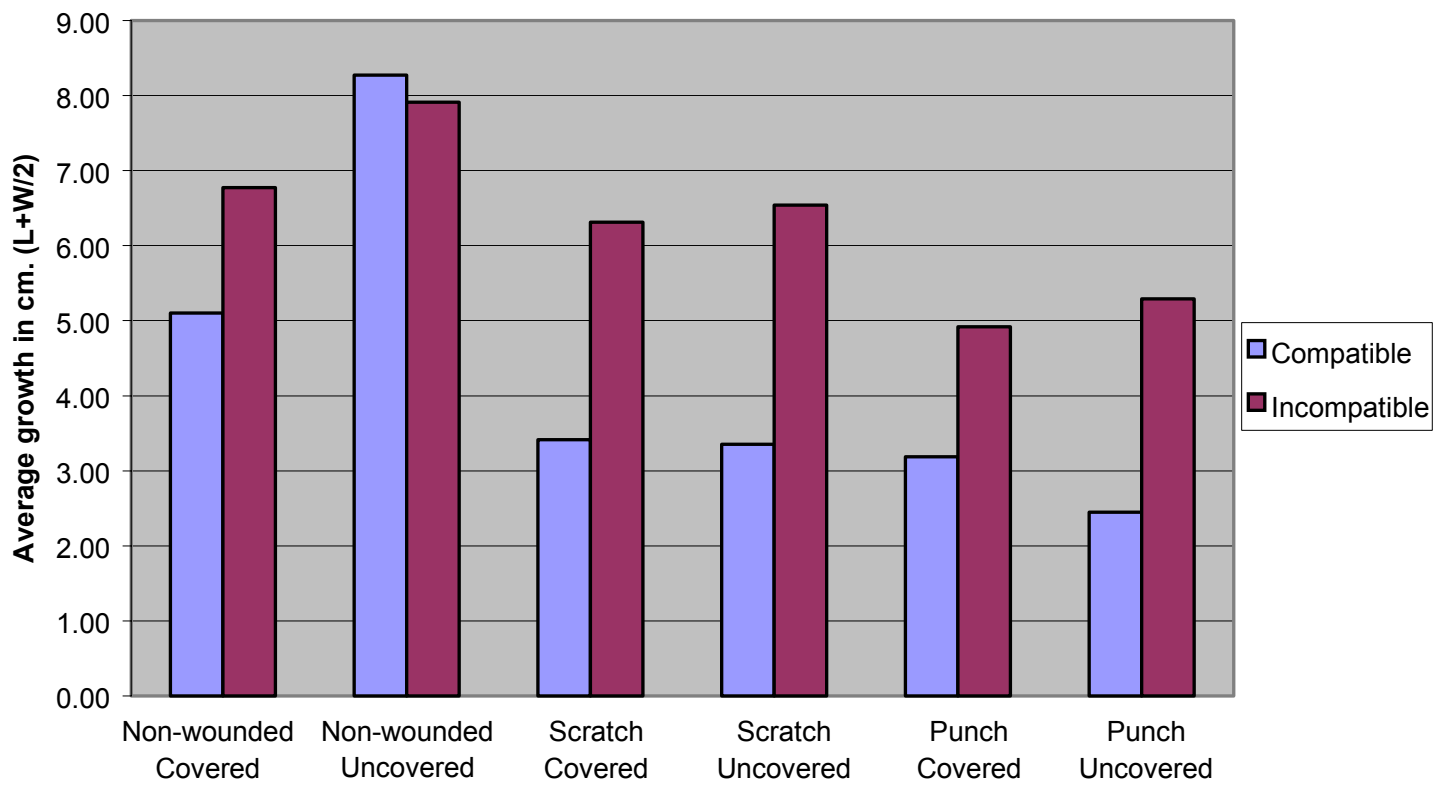

Figure 11. Average canker expansion from time of treatment $\left(\mathrm{T}_{0}\right)$ to second sample period $\left(\mathrm{T}_{2}\right)$. 


\section{Bark Sample Analysis (First Sample Period)}

The recovery of $\mathrm{OH}$ isolates provided evidence of hypovirus transmission. More $\mathrm{OH}$ isolates were recovered in the compatible than in the incompatible treatments, with the exception of the punch-uncovered method (Figs. $12 \& 13$ ). The increase in $\mathrm{OH}$ recovery was compensated by a corresponding decrease of orange-virulent $(\mathrm{OV})$ isolates. Further, brown-hypovirulent $(\mathrm{BH})$ treatment inoculum was recovered at a higher frequency when the cankers were covered, regardless of treatment.

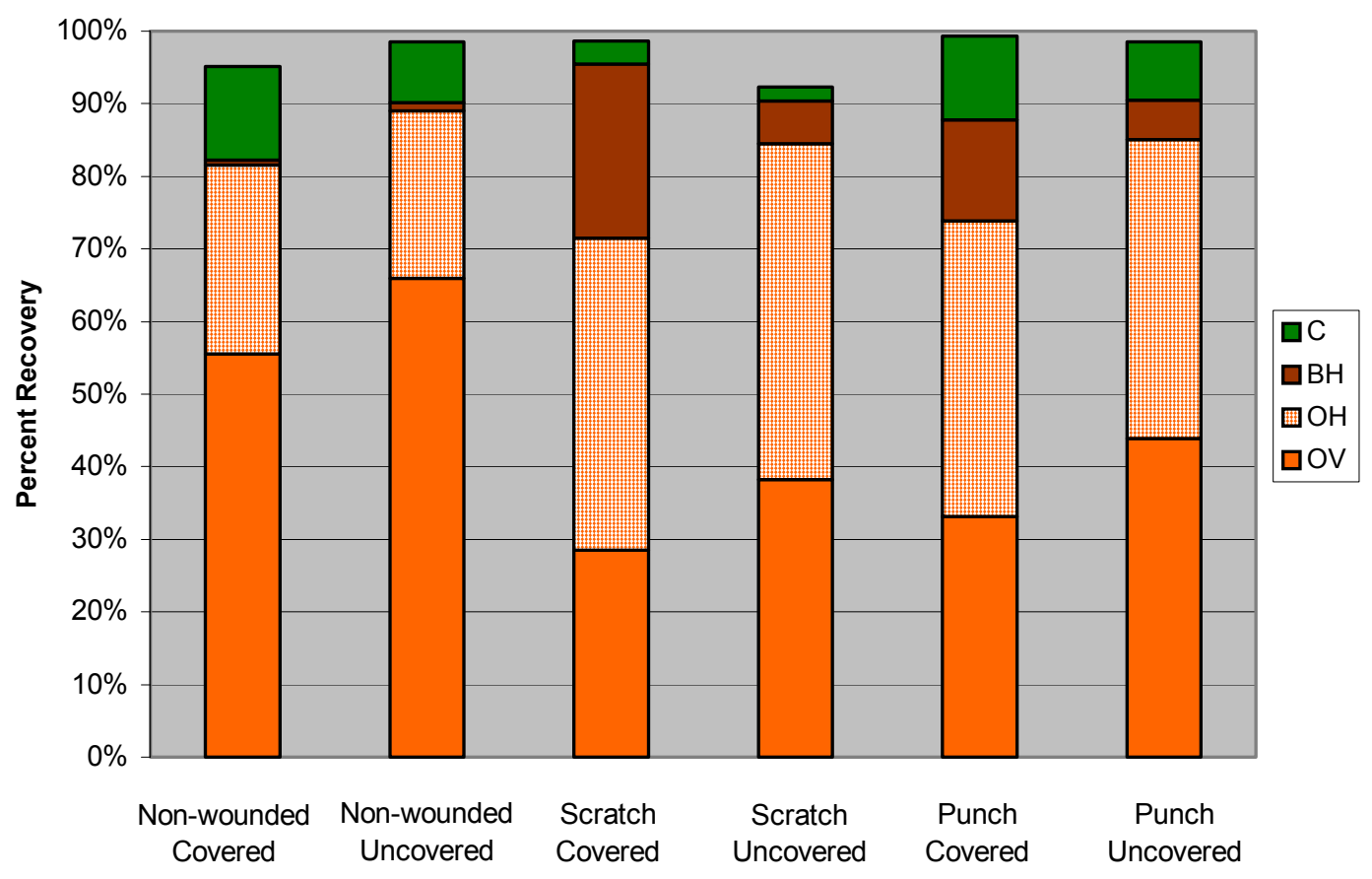

Figure 12. Percentage of orange-virulent (OV), orange-hypovirulent $(\mathrm{OH})$, brownhypovirulent $(\mathrm{BH})$, and non-Cryphonectria $(\mathrm{C})$ isolates recovered from compatible treatments sampled on November 15, 2002. 


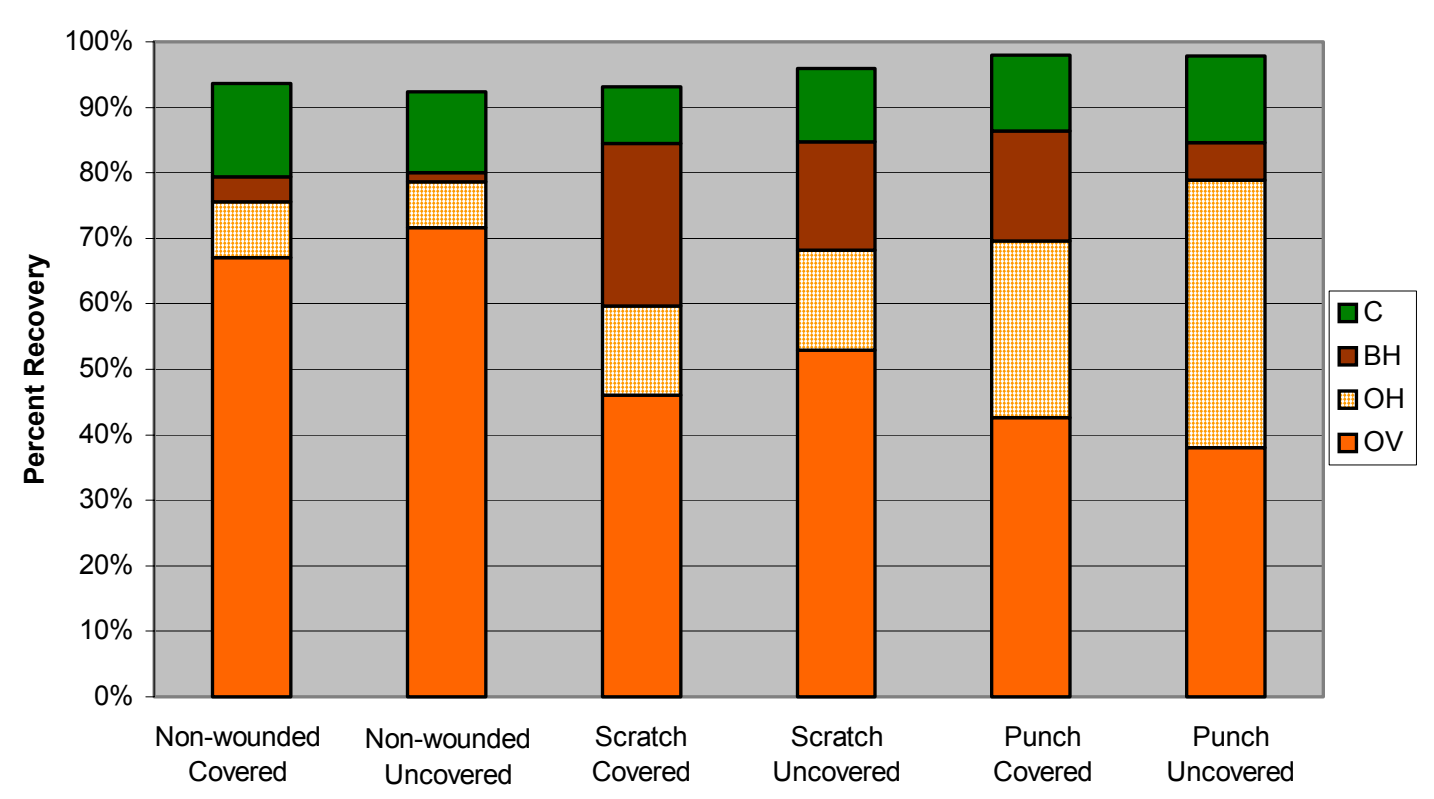

Figure 13. Percentage of orange-virulent $(\mathrm{OV})$, orange-hypovirulent $(\mathrm{OH})$, brownhypovirulent $(\mathrm{BH})$, and non-Cryphonectria $(\mathrm{C})$ isolates recovered from incompatible treatments sampled on November 15, 2002.

Comparable numbers of non-Cryphonectria organisms were isolated during the first sample period among the compatible and incompatible treatments, although there were slightly more non-Cryphonectria isolates recovered among the incompatible treatments.

\section{Bark Sample Analysis (Second Sample Period)}

During the second sample period, more $\mathrm{OH}$ isolates generally were recovered in the second sample from both the compatible and incompatible treatments, compared to the first sample (Figures $14 \& 15$ vs. $12 \& 13$ ). However, this change was very small among all treatments and not statistically significant. Like the first sample period, OV isolates were recovered more frequently in the non-wounded cankers than in the scratch 
or punch wounded cankers when challenged with either the compatible or incompatible treatment inoculum. Brown-hypovirulent treatment inoculum also was recovered more frequently in both the compatible and incompatible treatments during the second sample period when the cankers were covered. Furthermore, treatment inoculum was most frequently isolated from the scratch covered cankers.

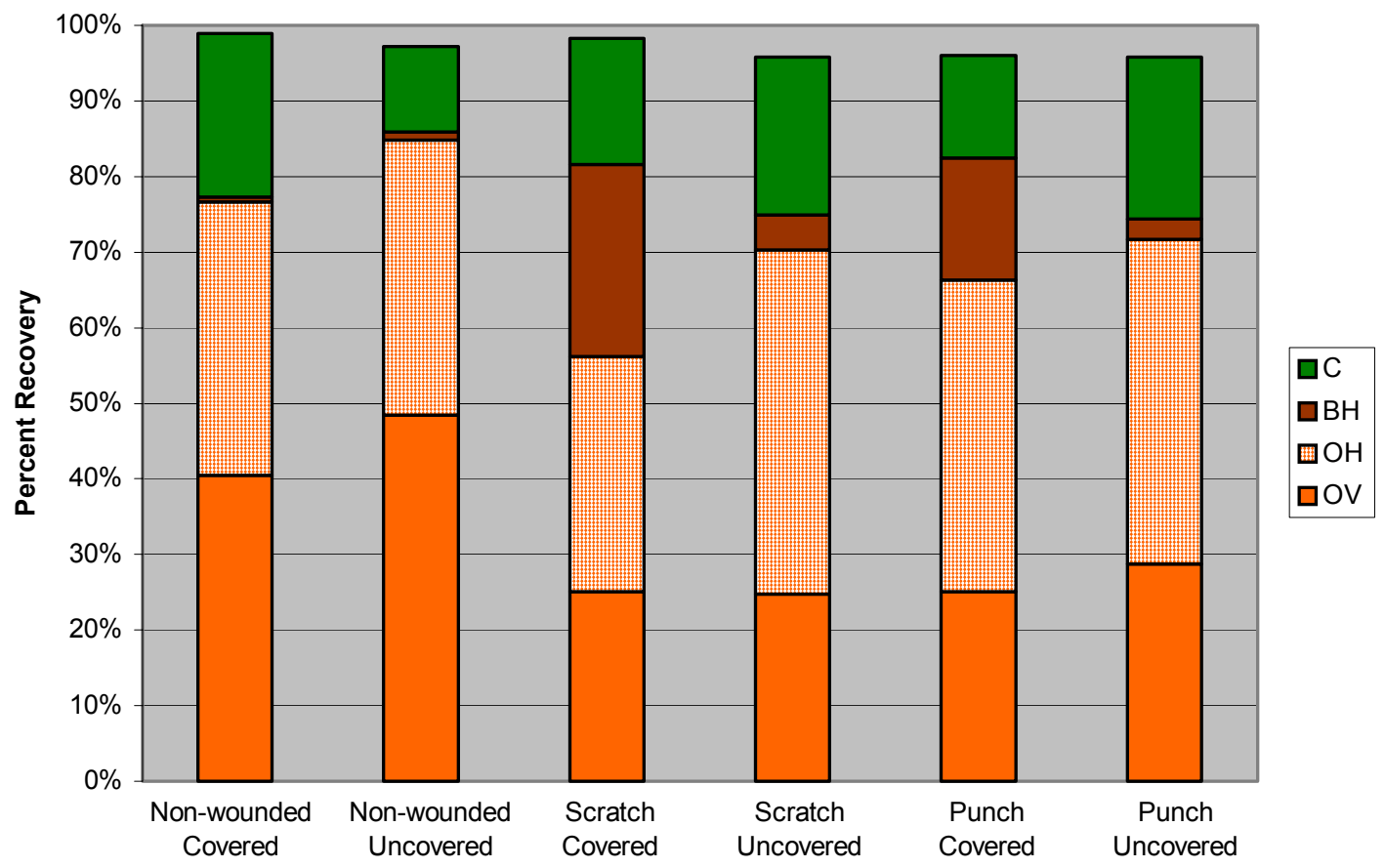

Figure 14. Percentage of orange-virulent $(\mathrm{OV})$, orange-hypovirulent $(\mathrm{OH})$, brownhypovirulent $(\mathrm{BH})$, and non-Cryphonectria $(\mathrm{C})$ isolates recovered from compatible treatments sampled on May 28, 2003.

Comparable numbers of non-Cryphonectria organisms were isolated during the second sampling period from the compatible and incompatible treatments. As in the first sample period, more non-Cryphonectria isolates were recovered from the incompatible treatments. 


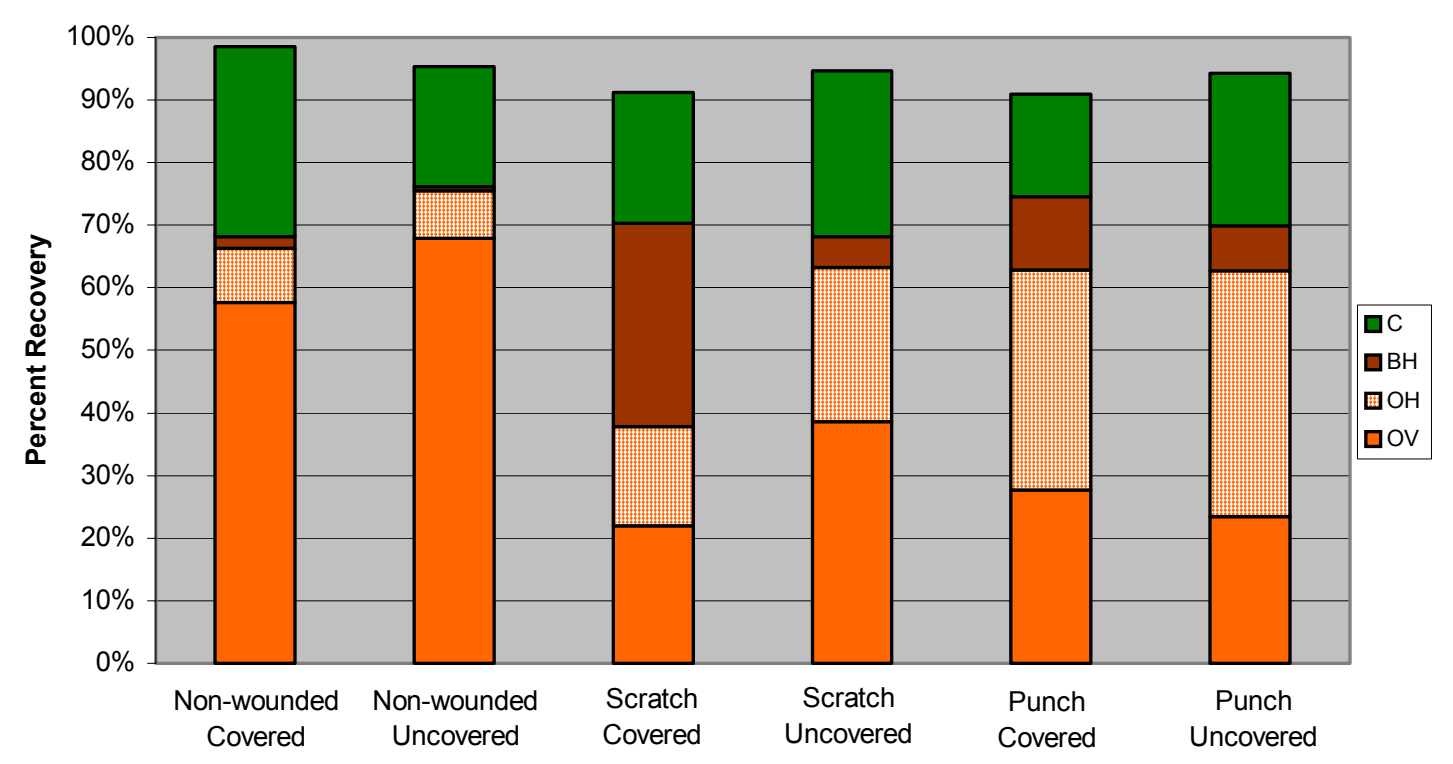

Figure 15. Percentage of virulent $(\mathrm{OV})$, orange-hypovirulent $(\mathrm{OH})$, brown-hypovirulent $(\mathrm{BH})$, and non-Cryphonectria $(\mathrm{C})$ isolates recovered from incompatible treatments sampled on May 28, 2003.

The following results were found to be common to both sample periods (Figures 12-15). A significantly greater number of $\mathrm{OH}$ isolates were recovered from cankers treated with compatible inoculum than those treated with incompatible inoculum $(\mathrm{P}>\mathrm{F}=$ $<0.0001)$. Significantly more $\mathrm{OH}$ isolates were recovered when cankers were wounded compared to non-wounded treatments $(\mathrm{P}>\mathrm{F}=<0.0001)$. Punching cankers resulted in the recovery of significantly more $\mathrm{OH}$ isolates than scratch wounding $(\mathrm{P}>\mathrm{F}=0.0046)$. Wounding cankers resulted in significantly greater recovery of $\mathrm{OH}$ isolates from the compatible than incompatible treatments $(\mathrm{P}>\mathrm{F}=0.0134)$. Punch-treating cankers also resulted in significantly better recovery of $\mathrm{OH}$ isolates when cankers were treated with incompatible compared to compatible inoculum $(\mathrm{P}>\mathrm{F}=0.0004)$. 
Brown-hypovirulent isolate recovery was significantly greater when cankers were treated with incompatible inoculum across all treatments $(\mathrm{P}>\mathrm{F}=0.0037)$ and, significantly more $\mathrm{BH}$ isolates were recovered in the fall than the spring $(\mathrm{P}>\mathrm{F}=0.0055)$. Covering cankers also resulted in recovery of significantly more $\mathrm{BH}$ isolates than uncovered cankers $(\mathrm{P}>\mathrm{F}=<0.0001)$. Further, wounding cankers yielded significantly more $\mathrm{BH}$ isolates than when cankers were not wounded $(\mathrm{P}>\mathrm{F}=<0.0001)$, with the scratch treatment yielding significantly greater recovery of $\mathrm{BH}$ isolates than the punch treatment $(\mathrm{P}>\mathrm{F}=0.0100)$. This resulted in a significant interaction between wounding and covering reflected in the greater recovery of $\mathrm{BH}$ isolates $(\mathrm{P}>\mathrm{F}=0.0016)$.

Recovery of the canker-inciting OV isolates was significantly greater when cankers were treated with incompatible inoculum, compared to treatments with compatible inoculum $(\mathrm{P}>\mathrm{F}=0.0055)$ with the first sample period yielding significantly more $\mathrm{OV}$ isolates than the second sample period $(\mathrm{P}>\mathrm{F}=0.0022)$. Also, there were significantly more OV isolates recovered from the non-wounded treatments than the wounded treatments $(\mathrm{P}>\mathrm{F}=<0.0001)$.

\section{Pre-treatment vs. Post-treatment Analysis}

Similar cultural results were obtained from November and May samples, when pre-treatment areas were compared to post-treatment areas of the canker (Figures $16-$ 19). Recovery of $\mathrm{OH}$ isolates from post-treatment areas was significantly higher than pre-treatment areas for all treatments $(\mathrm{P}>\mathrm{F}=<0.0001)$. Punch wounding cankers had a greater effect on $\mathrm{OH}$ isolate recovery when cankers were treated with incompatible inoculum in the pre-treatment areas of cankers. When cankers were treated with 
compatible hypovirulent inoculum, scratching had a greater effect on $\mathrm{OH}$ isolate recovery in pre-treatment areas $(\mathrm{P}>\mathrm{F}=0.0060)$.

Orange-virulent isolate recovery was significantly less frequent in the posttreatment area of the cankers than pre-treatment areas when they were treated with both compatible and incompatible inoculum $(\mathrm{P}>\mathrm{F}=<0.0001)$. Also, wounded treatments yielded significantly fewer OV isolates recovered from post-treatment areas of cankers, $(\mathrm{P}>\mathrm{F}=<0.0001)$ and this decrease corresponded to an increase in recovery of $\mathrm{OH}$ and $\mathrm{BH}$ isolates. The interaction between time (spring vs. fall) and area (pre vs. posttreatment) resulted in significantly more $\mathrm{OV}$ isolates being recovered in the fall than in the spring from pre-treatment areas of cankers $(\mathrm{P}>\mathrm{F}=0.0031)$.

Brown-hypovirulent treatment inoculum was recovered significantly more from the margins of cankers than the centers, for all treatments $(\mathrm{P}>\mathrm{F}=<0.0001)$. Also, coverings significantly increased the recovery of $\mathrm{BH}$ isolates from post-treatment areas $(\mathrm{P}>\mathrm{F}=0.0003)$. When considering the interaction between canker areas (pre vs. posttreatment) and wounding, there were significantly more $\mathrm{BH}$ isolates recovered from posttreatment areas than pre-treatment areas of cankers when they were wounded $(\mathrm{P}>\mathrm{F}=$ $<0.0001$ ) and scratch wounding yielded significantly more BH isolates than punchwounding $(\mathrm{P}>\mathrm{F}=0.0117)$.

Finally, non-Cryphonectria isolate recovery was similar among compatible and incompatible treatments, regardless of whether they were taken from pre-treatment or post-treatment areas, but the significance was not tested. 

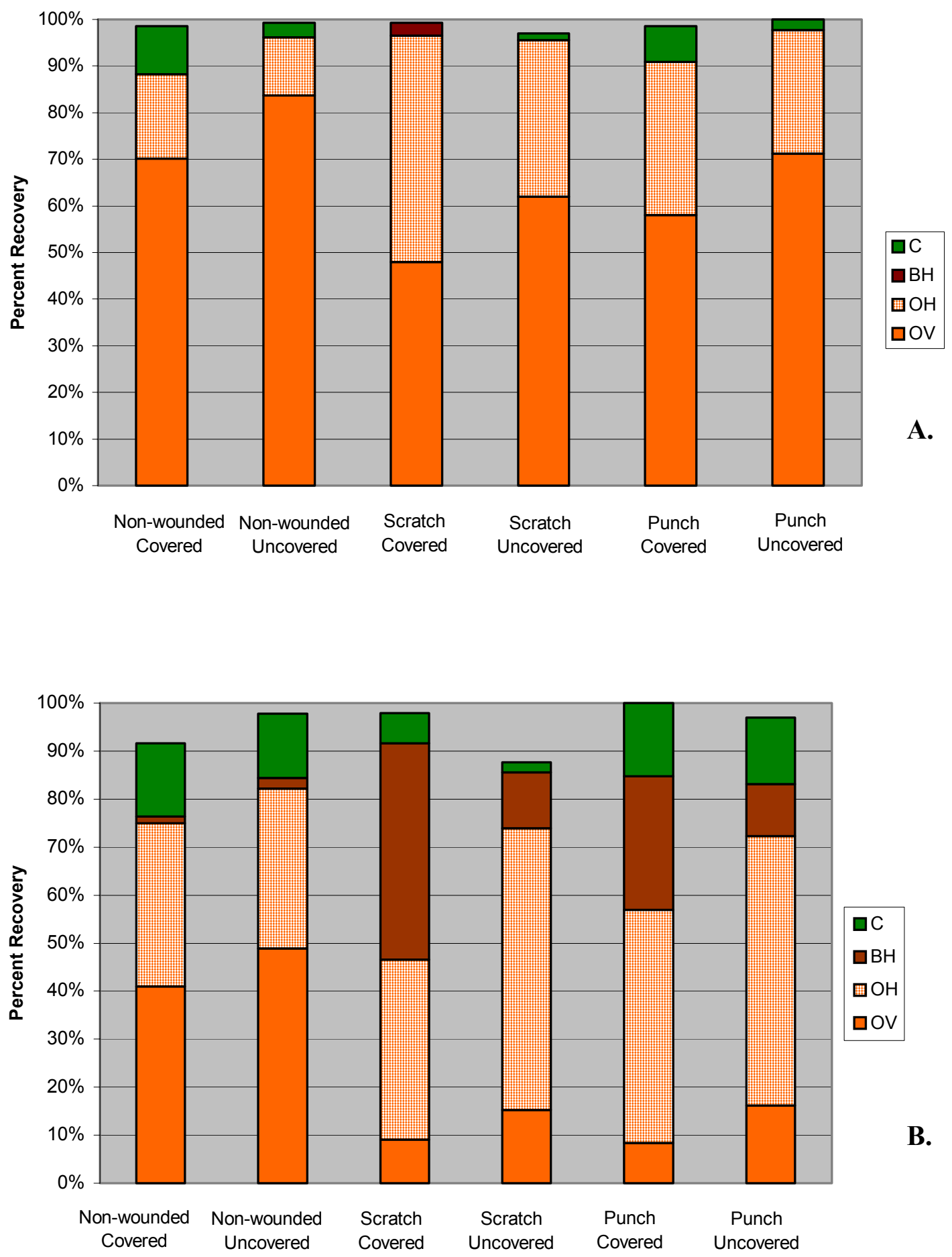

B.

Figure 16. Percentage of orange-virulent $(\mathrm{OV})$, orange-hypovirulent $(\mathrm{OH})$, brownhypovirulent $(\mathrm{BH})$, and non-Cryphonectria $(\mathrm{C})$ isolates recovered from compatible treatments following the November 2002 sample. Panel A, positions 13-24 (pretreatment). Panel B, positions 1-12 (post-treatment). 

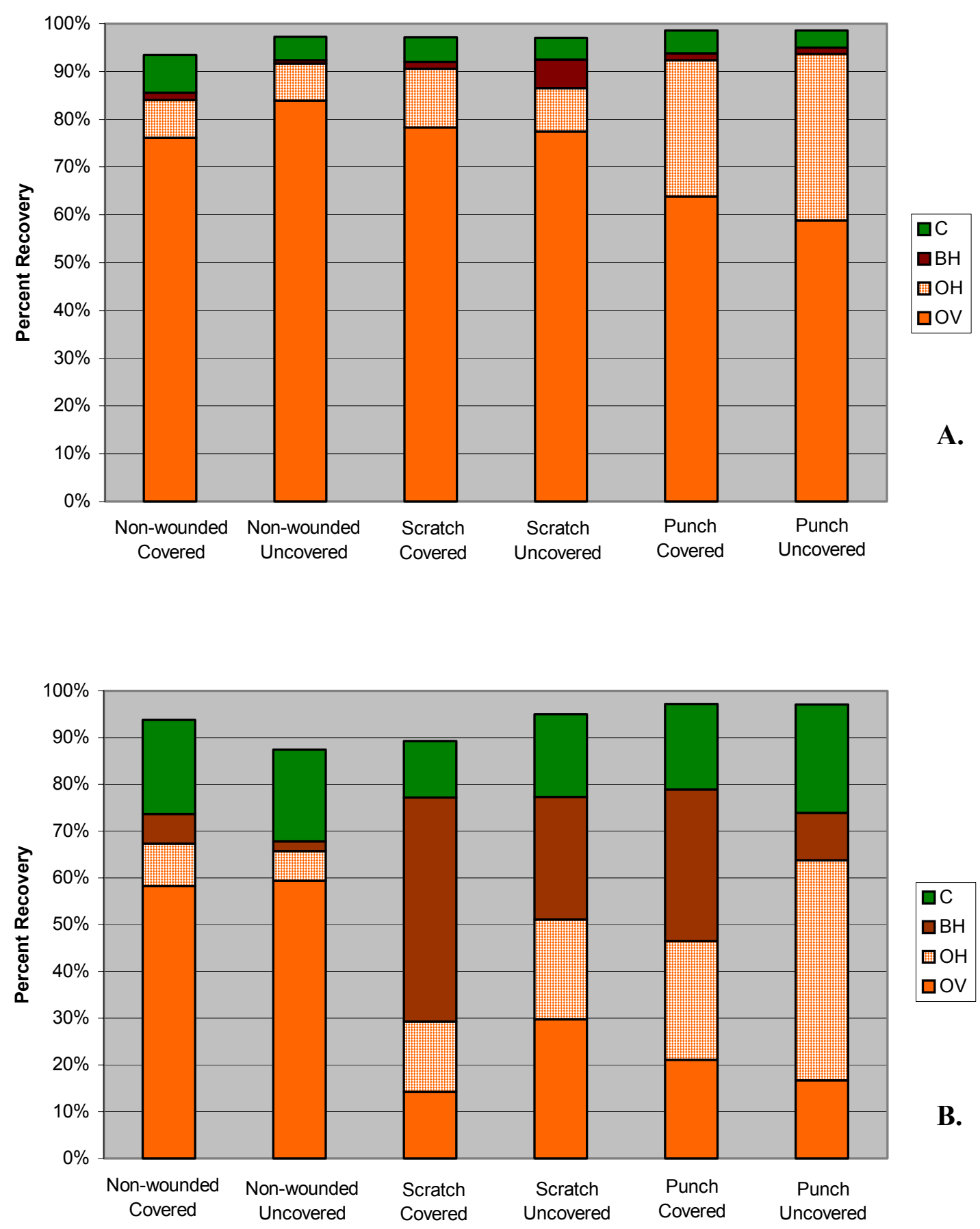

Figure 17. Percentage of orange-virulent $(\mathrm{OV})$, orange-hypovirulent $(\mathrm{OH})$, brownhypovirulent $(\mathrm{BH})$, and non-Cryphonectria $(\mathrm{C})$ isolates recovered from incompatible treatments following the November 2002 sample. Panel A, positions 13-24 (pretreatment). Panel B, positions 1-12 (post-treatment) 

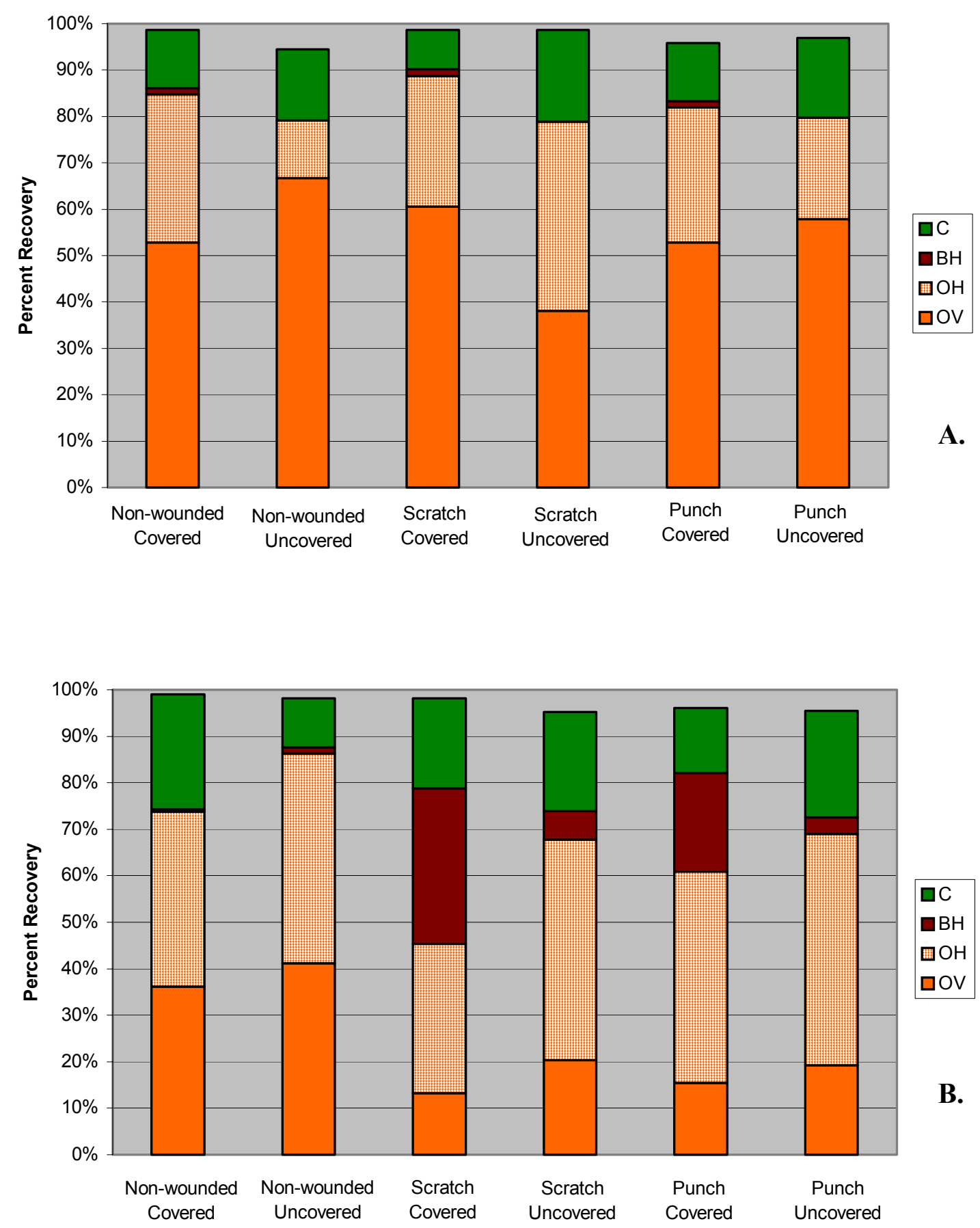

Figure 18. Percentage of orange-virulent $(\mathrm{OV})$, orange-hypovirulent $(\mathrm{OH})$, brownhypovirulent $(\mathrm{BH})$, and non-Cryphonectria $(\mathrm{C})$ isolates recovered from compatible treatments following the May 2003 sample. Panel A, positions 19-24 (pre-treatment). Panel B, positions 1-18 (post-treatment). 

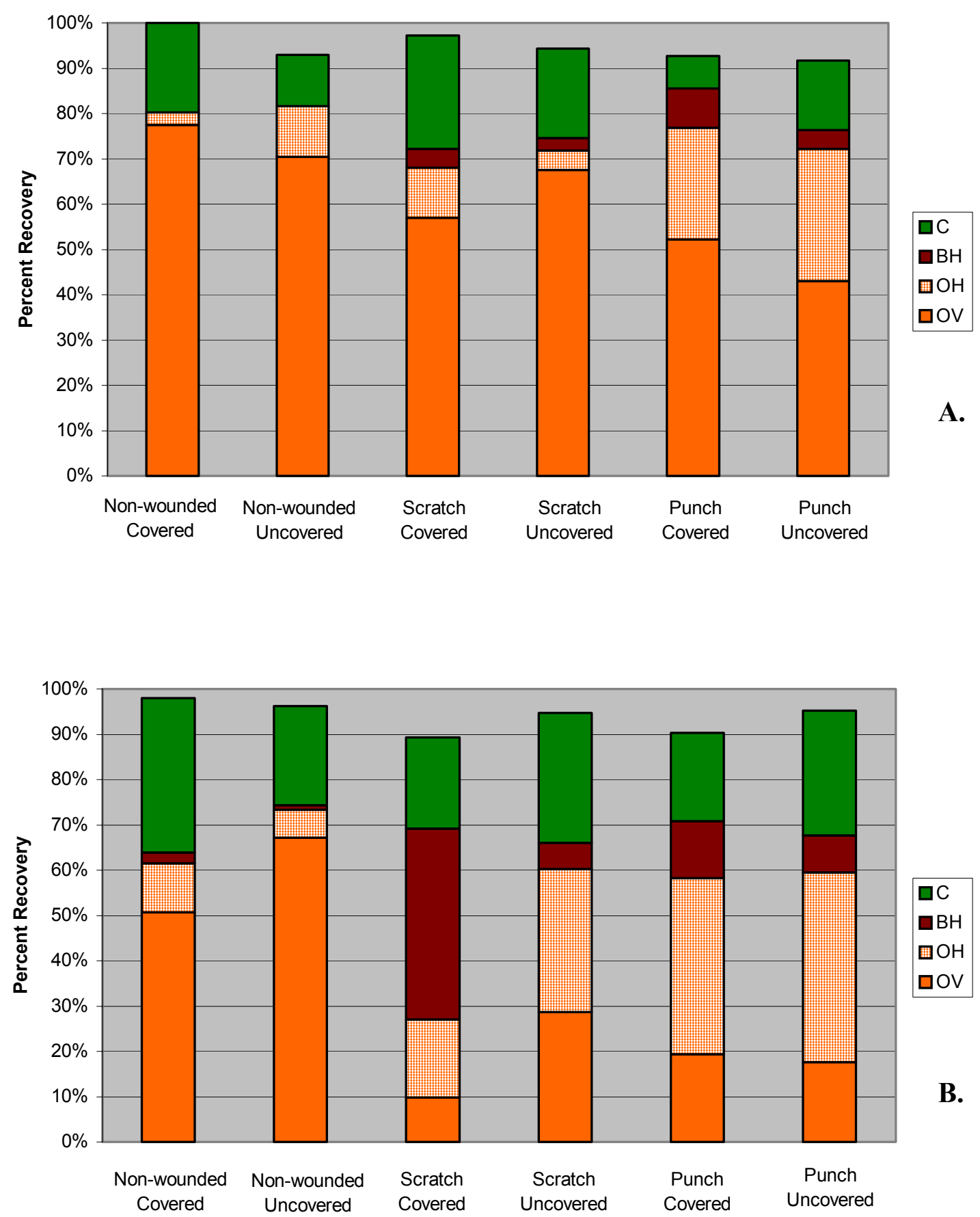

Figure 19. Percentage of orange-virulent $(\mathrm{OV})$, orange-hypovirulent $(\mathrm{OH})$, brownhypovirulent $(\mathrm{BH})$, and non-Cryphonectria $(\mathrm{C})$ isolates recovered from incompatible treatments following the May 2003 sample. Panel A, positions 19-24 (pre-treatment). Panel B, positions 1-18 (post-treatment). 


\section{Orange-Hypovirulent Isolate Pigmentation}

Throughout the course of this study, orange-hypovirulent isolates recovered from bark plugs expressed two levels of pigmentation. Isolates recorded as high-pigmented had an abundance of raised aerial hyphae and a bright orange pigmentation, whereas, low-pigmented isolates had little or no aerial hyphae and possessed very little pigmentation (Figure 20).
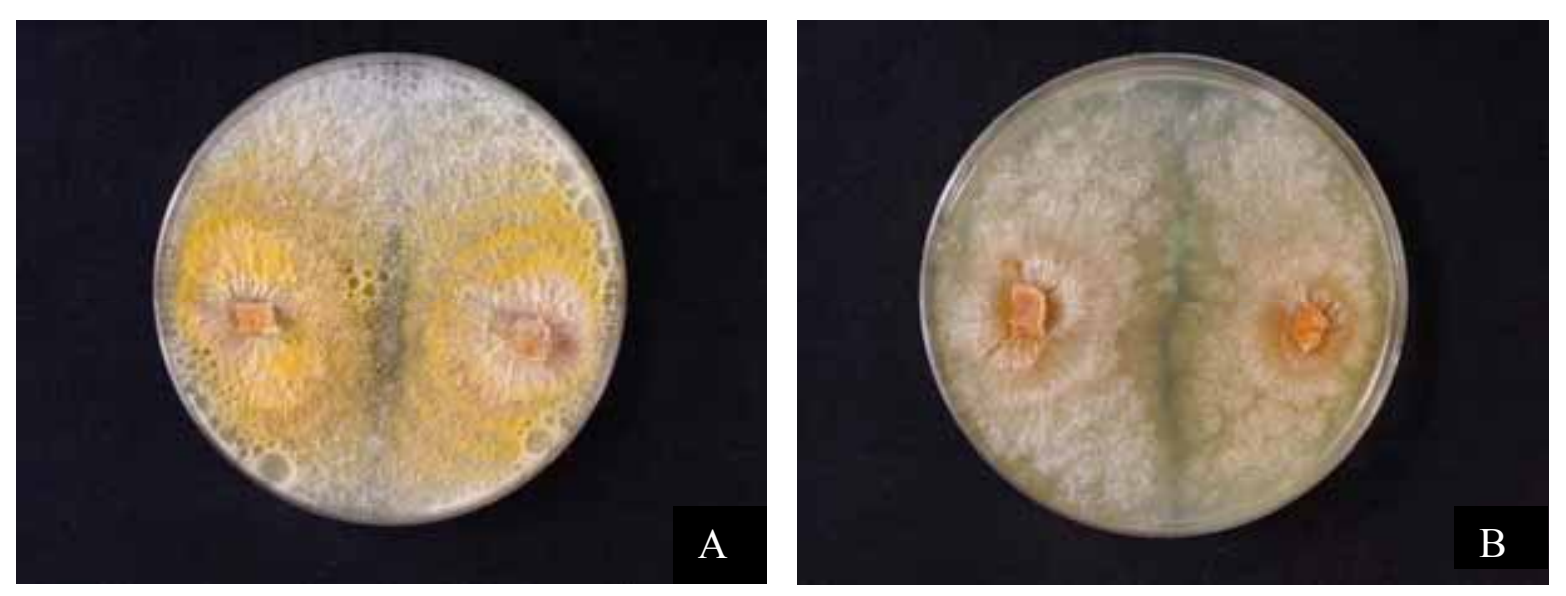

Figure 20. Morphologies of high (A) and low (B) pigmented orange-hypovirulent isolates.

Representative high and low pigmented isolates were selected at random and single spored to determine if pigmentations were stable among single-spore conidial colonies. In every case, a mass isolate, either high or low pigmented, yielded single spore colonies that were a mixture of high and low pigment types. However, subsequent single sporing of these colonies resulted in uniform pigmentation. That is, when a lowpigmented single spore colony from a high-pigmented mass isolate was single spored, it yielded all low-pigmented colonies. Conversely, a high-pigmented colony from a lowpigmented mass isolate yielded all high-pigmented colonies; the converse also was true. 
The stable high and low-pigmented orange-hypovirulent isolates that were the result of single sporing were tested numerous times for the presence of dsRNA by gel electrophoresis. Presence of bands indicated that both isolate types contained dsRNA, confirming that classifying them as hypovirulent throughout the study was correct (Figure 21).

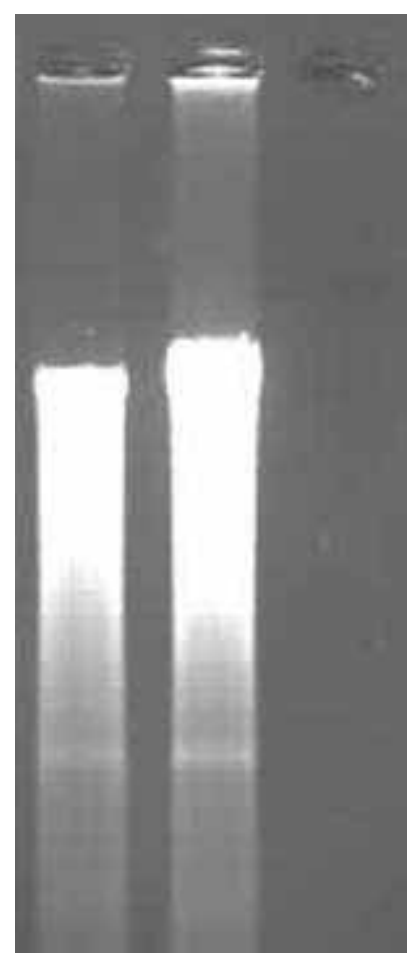

Figure 21. Agarose gel analysis indicating presence of dsRNA in high and lowpigmented isolates. From left to right, Lanes 1 and 2 represent dsRNA-containing isolates 12-T-17 (high) and 9-B-11 (low) respectively. Lane 3 represents a dsRNA-free virulent isolate.

An apple pathogenicity test also was conducted using the same isolates from above. In this test, six replications of Granny Smith apples were inoculated with the high and low-pigmented orange-hypovirulent isolates and a virulent isolate for comparison (Figure 22). In the apple pathogenicity test, the low-pigmented isolates were 
considerably less pathogenic than the high-pigmented isolate in every replicate. The high-pigmented isolates were comparably pathogenic to the virulent isolates (Figure 22).

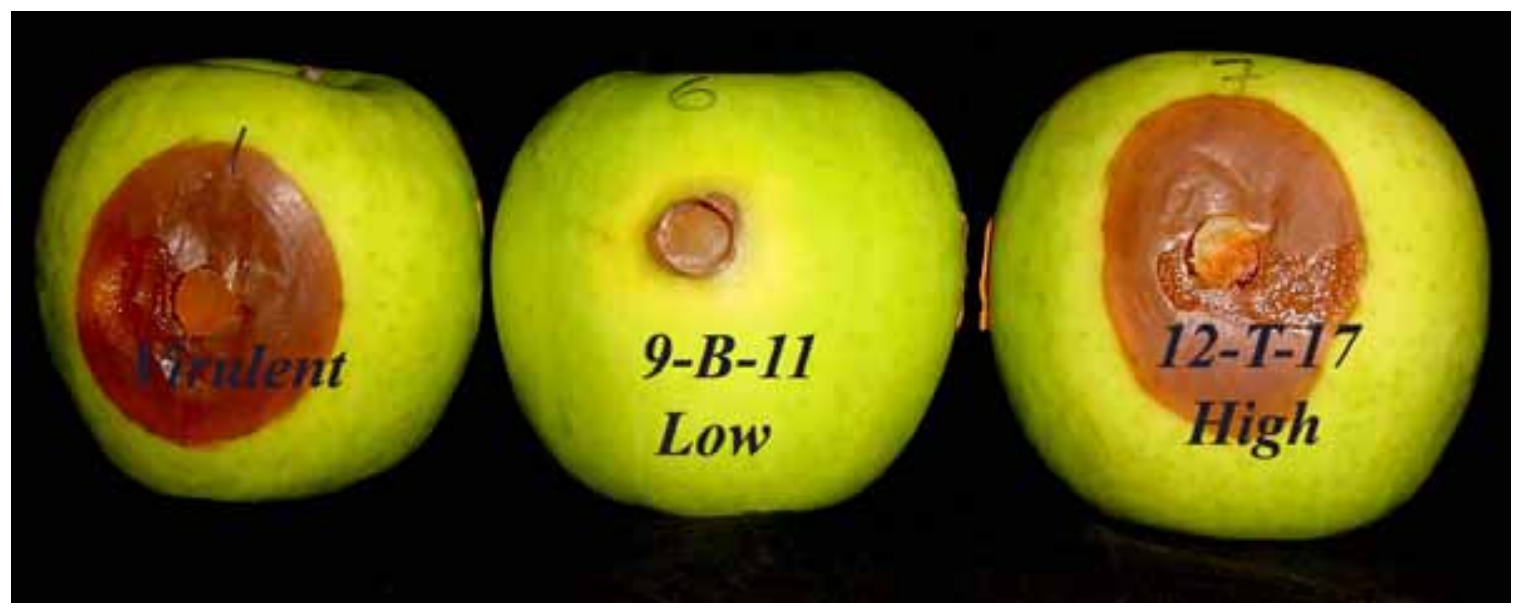

Figure 22. Results of apple pathogenicity test with a low pigmented (9-B-11), a highpigmented (12-T-17) and a virulent isolate.

\section{Single Sporing}

Single-spore colonies from bark pycnidia of treated cankers were evaluated by pigmentation and morphology to determine if one treatment yielded more hypovirulent conidia than another (Tables $1-4)$. In all treatments, orange-virulent $(\mathrm{OV})$ spores were recovered in higher frequencies than other spore types. For most pycnidia, over $90 \%$ of the conidia recovered were OV spores. During the November 2002 sample period (Tables 1 and 2), there were more OV spores recovered in the incompatible treatments than in the compatible treatments, with the exception of the non-wounded uncovered and scratch covered treatments. When $\mathrm{OH}$ spores were recovered, they weren't consistently associated with any particular treatment, although they were more likely to be recovered from treatments where wounding occurred. Brown-hypovirulent $(\mathrm{BH})$ spores, although 
recovered at a low frequency, were isolated more frequently from compatible than incompatible treatments.

During the May 2003 sample period (Tables 3 and 4), comparable numbers of OV spores were recovered from compatible $(93.8 \%)$ and incompatible treatments $(92.9 \%)$. The two exceptions were the punch-covered and punch-uncovered treatments. Similar to the November 2002 sample, OH spores were more likely to be recovered from wounded cankers. Finally, BH spores were recovered at a low frequency, similar to the November 2002 sample, and the majority of these spores came from the incompatible treatments when cankers were punched and left uncovered. 


\begin{tabular}{|c|c|c|c|c|c|}
\hline \multicolumn{3}{|c|}{ November 2002 Compatible } & \multirow[b]{2}{*}{$\mathrm{OH}$} & \multirow[b]{2}{*}{$\mathrm{BH}$} & \multirow{8}{*}{ Table 1} \\
\hline Treatment & Total Spores & OV & & & \\
\hline Non-Wounded Covered & 197 & $190(96 \%)$ & $2(1 \%)$ & $5(3 \%)$ & \\
\hline Non-Wounded Uncovered & 366 & $358(98 \%)$ & $0(0 \%)$ & $8(2 \%)$ & \\
\hline Scratch Covered & 238 & $237(99 \%)$ & $1(1 \%)$ & $0(0 \%)$ & \\
\hline Scratch Uncovered & 189 & $175(92 \%)$ & $9(5 \%)$ & $5(3 \%)$ & \\
\hline Punch Covered & 301 & $300(99 \%)$ & $1(1 \%)$ & $0(0 \%)$ & \\
\hline Punch Uncovered & 295 & $278(94 \%)$ & $17(6 \%)$ & $0(0 \%)$ & \\
\hline \multicolumn{3}{|c|}{ November 2002 Incompatible } & & & \multirow{8}{*}{ Table 2} \\
\hline Treatment & Total Spores & OV & $\mathrm{OH}$ & $\mathrm{BH}$ & \\
\hline Non-Wounded Covered & 408 & $393(96 \%)$ & $15(4 \%)$ & $0(0 \%)$ & \\
\hline Non-Wounded Uncovered & 225 & $225(100 \%)$ & $0(0 \%)$ & $0(0 \%)$ & \\
\hline Scratch Covered & 233 & $232(99 \%)$ & $0(0 \%)$ & $1(1 \%)$ & \\
\hline Scratch Uncovered & 380 & $377(99 \%)$ & $0(0 \%)$ & $3(1 \%)$ & \\
\hline Punch Covered & 363 & $337(93 \%)$ & $26(7 \%)$ & $0(0 \%)$ & \\
\hline Punch Uncovered & 480 & $430(89 \%)$ & $50(11 \%)$ & $0(0 \%)$ & \\
\hline
\end{tabular}

\section{May 2003 Compatible}

\begin{tabular}{|c|c|c|c|c|}
\hline Treatment & Total Spores & OV & $\mathrm{OH}$ & $\mathrm{BH}$ \\
\hline Non-Wounded Covered & 343 & 335 (98\%) & $4(1 \%)$ & $4(1 \%)$ \\
\hline Non-Wound Uncovered & 498 & $497(99 \%)$ & $1(1 \%)$ & $0(0 \%)$ \\
\hline Scratch Covered & 290 & $290(100 \%)$ & $0(0 \%)$ & $0(0 \%)$ \\
\hline Scratch Uncovered & 315 & $266(85 \%)$ & $49(16 \%)$ & $0(0 \%)$ \\
\hline Punch Covered & 163 & $113(69 \%)$ & $50(31 \%)$ & $0(0 \%)$ \\
\hline Punch Uncovered & 150 & $150(100 \%)$ & $0(0 \%)$ & $0(0 \%)$ \\
\hline
\end{tabular}

\section{May 2003 Incompatible}

\begin{tabular}{|c|c|c|c|c|}
\hline Treatment & Total Spores & OV & $\mathrm{OH}$ & $\mathrm{BH}$ \\
\hline Non-Wounded Covered & 328 & $326(99 \%)$ & $2(1 \%)$ & $0(0 \%)$ \\
\hline Non-Wounded Uncovered & 310 & $309(99 \%)$ & $1(1 \%)$ & $0(0 \%)$ \\
\hline Scratch Covered & 246 & 245 (99\%) & $1(1 \%)$ & $0(0 \%)$ \\
\hline Scratch Uncovered & 239 & $227(95 \%)$ & $12(5 \%)$ & $0(0 \%)$ \\
\hline Punch Covered & 141 & $133(94 \%)$ & $8(6 \%)$ & $0(0 \%)$ \\
\hline Punch Uncovered & 248 & $165(66 \%)$ & $61(26 \%)$ & $22(8 \%)$ \\
\hline
\end{tabular}

Tables 1 - 4. Orange-virulent $(\mathrm{OV})$, orange-hypovirulent $(\mathrm{OH})$, and brown-hypovirulent (BH) conidia recovered from November 2002 and May 2003 sampling periods.

Percentages are indicated in parenthesis. 


\section{Sectoring}

Sectoring from bark plug colonies was common to all treatments and was noted more frequently during the initial culturing of the bark plugs on GYE/A than after transfer to PDA (Figures 23A and 23B). Further, sectoring was more common in treatments that involved wounding. Table 5 shows types of sectors noted from all samples taken during the second sample period when colonies were subcultured to PDA plates. There were more orange-hypovirulent $(\mathrm{OH})$ sectored isolates found in the wounded treatments, the one exception being the non-wounded compatible treatment. Sectoring appeared independent of compatibility type but certain types of sector combinations appeared more common to some compatible or incompatible treatments. For example, the combination of $\mathrm{OV} / \mathrm{BH}$ was most common in the incompatible wounded treatments and least common in the compatible treatments (Table 5). 

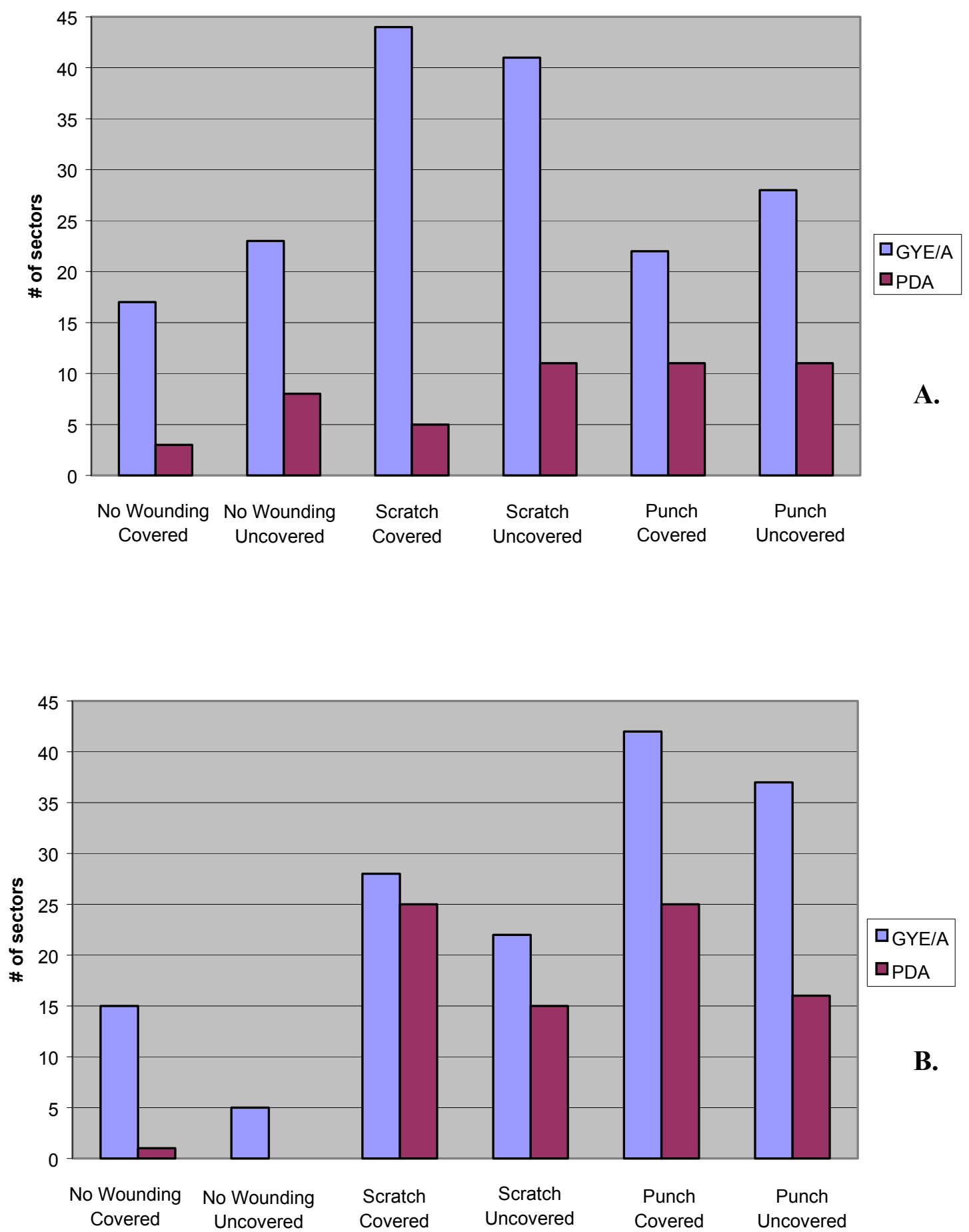

Figure 23. Differences in number of colony sectors on GYE/A and PDA media. A. represents compatible treatments while B. represents incompatible treatments. Each treatment represents the number of sectors observed from 286 isolations. 


\begin{tabular}{cccc}
\hline \multicolumn{3}{c}{ Sector Types } & \\
\hline Types & OH / BH & OV / OH & OV / BH \\
\hline & & & \\
Compatible Treatments & & & \\
Non-Wounded Covered & 0 & 2 & 1 \\
Non-Wounded Uncovered & 0 & 7 & 1 \\
Scratch Covered & 2 & 2 & 1 \\
Scratch Uncovered & 4 & 5 & 1 \\
Punch Covered & 5 & 5 & 1 \\
Punch Uncovered & 3 & 6 & 2 \\
& & & \\
Incompatible Treatments & & & \\
Non-Wounded Covered & 1 & 0 & 0 \\
Non-Wounded Uncovered & 0 & 0 & 0 \\
Scratch Covered & 9 & 4 & 11 \\
Scratch Uncovered & 0 & 4 & 11 \\
Punch Covered & 13 & 1 & 10 \\
Punch Uncovered & 6 & 6 & 4
\end{tabular}

Table 5. Sectoring combinations isolated from the May 2003 sampling period. Each treatment represents the number of sector types observed from 286 isolations

\section{dsRNA Extraction Analysis}

The presence of dsRNA was used throughout the course of this study as a method to verify that the isolates designated as hypovirus-infected contained dsRNA. Random isolates representating $\mathrm{OV}, \mathrm{OH}$ and $\mathrm{BH}$ morphologies were selected while analyzing bark plug cultures from the first and second sample periods. The results of one representative dsRNA extraction from selected bark plug isolates of $C$. parasitica are shown in Figure 24. Of the $20 \mathrm{OH}$ and $10 \mathrm{BH}$ isolates analyzed, all isolates yielded bands in gel electrophoresis. Conversely, the 9 OV isolates tested lacked dsRNA. 


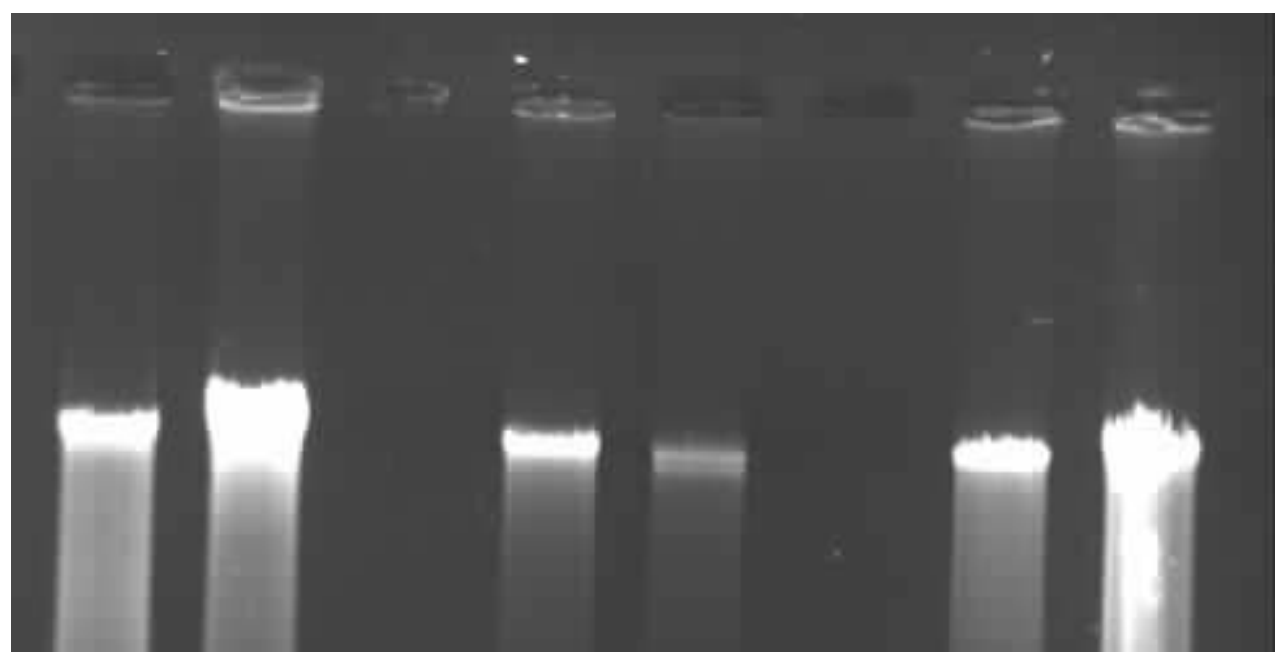

Figure 24. Agarose gel analysis indicating presence or absence of dsRNA. From left to right, lanes 1, 2, 7 and 8 represent dsRNA-containing orange-hypovirulent isolates, lanes 4 and 5 represent dsRNA-containing brown-hypovirulent isolates and lanes 3 and 6 represent dsRNA-free orange-virulent isolates. 


\section{Discussion}

Various methods have been used to introduce hypovirulent inoculum into natural and artificially established cankers on chestnut. The long-standing protocol for canker treatment is the margin-punch method developed by Grente and Berthelay-Sauret (1978), where agar plugs containing hypoviruses are applied to punch-wounds made at the canker margin. This method has been used effectively in many field studies but it is time consuming and labor intensive. Other means of delivering inoculum into cankers have included the use of hypovirulent bark patches and direct conidial sprays (Jaynes and DePalma, 1982; Hobbins, 1985; Balbalian, 1998). The punch treatment also has been used to deliver mixtures of hypovirulent isolates in some studies (Jaynes and Elliston, 1980; Shain and Miller, 1992).

Some conclusions about canker treatment efficacy can be drawn from tests even though the treatments were applied for other reasons. Double (1982) challenged artificially established cankers with individual hypovirulent isolates or mixtures of hypovirulent isolates using the margin-punch treatment method described by Grente and Berthelay-Sauret (1978). Although not the focus of his study, transmission of hypoviruses to the virulent thallus occurred at a high rate. However, he acknowledged that methods of establishing hypovirulent inoculum might be an obstacle to the success of hypovirulence as biological control.

Jaynes and Elliston (1980) used Grente's punch treatment method to apply mixtures of hypovirulent strains of $C$. parasitica and concluded that combining strains is an effective strategy for controlling individual virulent cankers. The purpose of using mixtures of hypovirulent strains is to overcome the barriers imposed by the system of 
vegetative incompatibility. These mixtures were highly effective in controlling canker expansion when compared to inoculating cankers with individual strains. However, the minimum number of strains needed to adequately control canker growth was not determined and probably depends on the vegetative compatibility type of the canker inciting strain and the inoculum used for treatment.

Shain and Miller (1992) also employed a modification of Grente's canker treatment method to evaluate hypovirus colonization of the fungal thallus. They introduced hypovirulent inoculum in a single cork-borer wound at the base of cankers and found that $100 \%$ of the canker margin was converted by a vegetatively compatible hypovirulent strain three weeks after treatment.

Jaynes and DePalma (1982) treated natural infections with mixtures of hypovirulent isolates by using both Grente's method of introduction and delivering conidia from a backpack mist blower. Even though their study was designed to track natural spread from one plot to another, the authors concluded that challenging preexisting cankers with mixtures of hypovirulent isolates is a viable method of treating cankers and can overcome some of the compatibility barriers. The spray technique used in their study was difficult to analyze because sprays and slurries were used on the same stems and often in the same canker. Nevertheless, they concluded that the use of hypovirulent mixtures could prolong survival of American chestnut stems.

Hobbins (1985) introduced hypovirulent treatment inoculum via chestnut bark patches. His objective was to provide a persistent source of hypovirulent inoculum as might be provided naturally by a hypovirulent infection. He inoculated sterilized bark patches in the laboratory with hypovirulent strains and mounted the fully colonized bark 
patches $10 \mathrm{~cm}$ above the cankers on study trees. He discovered that this treatment method was useful in delivering hypovirulent inoculum, particularly when the bark patch inoculum source was vegetatively compatible with the artificially initiated cankers. This method was intended to simulate natural sources of inoculum without wounding the cankers. Balbalian (1998) also used bark patches as a means of delivering hypovirulent treatment inoculum. Her study was designed to measure the relationship of vic gene differences to hypovirus acquisition from hypovirulent bark patches to artificially established cankers, all of known vegetative compatibility types. In her study, the bark patches were a less effective means of hypovirus delivery, when compared to results obtained by Hobbins. With the exception of the bark patch and conidial spray studies, two non-invasive procedures, almost all tests have involved a punch wound treatment to introduce a variety of different treatment inoculum.

In contrast to other tests, my study was specifically designed to evaluate and compare methods of introducing hypoviruses into cankers. The methods included two invasive treatments, one that consisted of wounding the canker with a sharp blade prior to treatment and a second where a series of punch wounds were made around the canker perimeter prior to hypovirus application, the procedure used by Grente. A third, noninvasive method involved application of the treatment inoculum by painting the canker surface. To determine whether a protective cover influenced treatment effectiveness, half of the cankers were covered for 18 days with an absorbent pad. Further, the use of vegetatively compatible and incompatible strains allowed for an evaluation of whether the treatment method could overcome barriers to hypovirus transmission that are imposed by the system of vegetative incompatibility. Prior to establishment of the study plot, 
there were concerns about dissemination of the treatment inoculum and how that might confound the results obtained in this study. Therefore, trees treated with compatible hypovirulent inoculum were separated physically, within the same clearcut area, from trees that were treated with incompatible hypovirulent inoculum. Also, other safeguards were implemented to prevent mixing of bark samples and cross contamination during the sampling periods. Cankers in different treatment regimes were sampled with different bone marrow biopsy instruments. In addition, the bone marrow biopsy instruments were sterilized in the field prior to sampling each canker. These safeguards, along with the design of the experiment presumably prevented any inaccurate representation of the results or cross contamination.

This study clearly demonstrates that wounding the canker prior to treatment greatly enhances the transmission of hypoviruses. Acquisition of hypovirus, evidenced by recovery of orange-hypovirulent isolates, was confirmed in all wounded treatments. Significantly more orange-hypovirulent isolates were recovered from wounded cankers compared to non-wounded treatments. Initially, it was hypothesized that the greater amount of wounding, which occurred when cankers were scratched, would promote more hypovirus acquisition than when cankers were punch wounded at the margin. However, this was not the case as punch-wounded treatments resulted in significantly better hypovirus acquisition than scratch-wounded treatments. One possible explanation for this finding is that punch wounds typically are deeper allowing for interaction between virulent and hypovirulent hyphae within multiple bark layers, thus affording more opportunities for anastomosis. Another explanation could be that the treatment inoculum desiccates quicker when cankers are scratch wounded as opposed to punch wounded prior 
to treatment. Also, the punch wounds were located at the active growing point of hyphae, an area not specifically targeted by the scratch wounding technique. The deep punch wounds created by Grente's canker treatment procedure may be the key to successful anastomosis and subsequent hypovirus acquisition.

Although hypovirus transmission was achieved most successfully when the punch treatment procedure was employed, recovery of the brown-hypovirulent treatment inoculum was best in the scratch-wounded treatments. The most plausible explanation for this finding is that the abundant number of scratch wounds provided more niches to trap the treatment inoculum over the entire surface of the canker. This technique may have permitted the treatment inoculum the opportunity to grow and become established, more so than with other treatments. Presumably, the treatment inoculum successfully infected the bark, as any superficial inoculum that might have remained from the treatment application would have been eliminated by the 14-minute bark plug sterilization procedure that was used prior to culture. Covering the cankers also contributed to the recovery of the brown-hypovirulent treatment inoculum, regardless of treatment. Presumably, the coverings protected the inoculum from the drying effects of the wind and washing aspect of the rain. Enhanced moisture levels provided by the coverings also may have afforded the treatment inoculum an opportunity to grow into the dead bark associated with the cankers. Since canker coverings were removed 18 days after treatment, the increased recovery of the treatment inoculum resulted after a relatively short period of protection. When canker coverings were removed, there were no visible differences in the appearance of covered versus uncovered cankers. It was hypothesized that canker coverings would provide greater moisture levels and thereby 
increase survival of, or infection by, the treatment inoculum. Data (Figures 12 - 15) support this hypothesis. However, the enhanced moisture levels created by the canker coverings do not appear to be critical to the establishment of hypoviruses or the success of treatment, even though they did enhance recovery of the treatment inoculum. Coverings may have restricted the activity of potential insect vectors. However, vectors may not be necessary when copious amounts of treatment inoculum are introduced onto cankers, as was done in this study. Further, the effect of wind, rain splash and stem flow is eliminated when the canker coverings are in place. The question as to whether canker coverings are useful in treatment success is unclear. In this test, coverings were important for greater recovery of the treatment inoculum, but not so much so for hypovirus acquisition. Canker coverings were only in place for 18 days, therefore, there may be other situations where coverings could be more important.

The design of the experiment prevented a specific estimate of the time frame in which virulent canker-inciting isolates acquired hypovirus. Because a 12-week interval existed between treatment and the first sampling period, the rapidity of hypovirus acquisition is unknown. To assess this, bark samples could be taken almost immediately after treatment and then at regular intervals. If brown-hypovirulent treatment isolates are recovered in significant numbers soon after treatment, the implication is that establishment and successful anastomosis is a longer-term process. However, if orangehypovirulent isolates were recovered soon after challenge, then anastomosis and hypovirus acquisition is rapid. However, the rapidity of anastomosis and hypovirus acquisition could depend on where anastomosis occurs and if hypovirus is found solely in newly formed hyphae. If only new hyphae contain hypoviruses, one would expect 
immediate recovery of orange-hypovirulent isolates in areas of the canker where mycelial growth is most active. Shain and Miller (1992) found that hypovirus acquisition occurs fairly rapidly, within three weeks, even though they used a hypovirus delivery method that differed from mine. Since the isolation results from my study were similar between the first and second sampling periods (Figures $12-15$ ), an increase in hypovirus acquisition was not noted. This implies that once hypovirus acquisition occurs it remains fairly constant, thus supporting the findings of Shain and Miller. Hobbins (1985) showed that treating cankers with vegetatively compatible hypovirulent isolates resulted in over one-half of the isolates recovered being hypovirulent, ten weeks after hypovirus challenge. Hobbins' results and the data reported by Shain and Miller (1992) are similar to the findings from my study that hypovirus acquisition occurs within weeks, rather than months.

The phenomenon of vegetative incompatibility in $C$. parasitica has received considerable attention because of its potential to restrict the transmission of hypoviruses as biological control agents (Huber, 1996). When strains of C. parasitica are vegetatively incompatible, virus transmission between isolates (horizontal transmission) is restricted (Anagnostakis, 1977; Milgroom and Cortesi, 2004). Vegetative incompatibility is a self / non-self recognition system that results in programmed cell death when cells of incompatible individuals attempt to anastomose (Milgroom and Cortesi, 2004). My study reemphasized the importance of vegetative compatibility to the transmission of hypoviruses. Based on data from several studies, vegetative incompatibility may be one of the most limiting factors to hypovirus transmission (Jaynes and Elliston, 1980; Hobbins, 1985; Shain and Miller, 1992; Balbalian, 1998). However, 
Milgroom and Cortesi (2004) state that the significance of vegetative incompatibility inhibiting transmission of hypoviruses in field studies may be overestimated. Milgroom and Cortesi (2004) claim that vegetative incompatibility alone is a poor predictor of biological control and that an integrated approach to control of C. parasitica seems more logical.

Vegetative incompatibility played a significant role in my study. When treatments were vegetatively compatible, regardless of the treatment procedure, they yielded significantly more orange-hypovirulent isolates than incompatible treatments. This study, like other field studies (Hobbins, 1985; Balbalian, 1998), confirmed that conversion can occur when strains are vegetatively incompatible, albeit at significantly lower rates.

Vegetative incompatibility in C. parasitica is controlled by at least six unlinked vic loci, with two alleles at each locus (Cortesi and Milgroom, 1998). The importance of specific vic genes to the transmission of hypoviruses was examined by Huber (1996) and Balbalian (1998). Huber (1996) examined the effects of specific vic genes on hypovirus transmission in vitro. He found that hypovirus transmission depends on the vegetative incompatibility genotype of both the hypovirulent donor and virulent recipient fungal strains. Further, he showed that the number of heteroallelic vic genes is not as important as the heteroallelic loci existing between interacting strains. Allelic differences (heteroallelism) at loci vic1, vic2 and vic3 strongly affect hypovirus transmission, whereas heteroallelism at vic4 and vic5 have little known effect on hypovirus transmission (Huber, 1996). Balbalian's (1998) study examined the effects of vegetativeincompatibility loci on horizontal transmission in a forest setting. She confirmed 
Huber's findings and found that hypovirus transmission was limited in part by the restrictions imposed by vic genes between the donor and recipient strains.

The vic gene differences of the three isolates used in my study were not known, posing some limitations to the interpretation of the results. Before the field experiment was established, preliminary studies on PDA showed that hypovirus transmission readily occurred between isolate 80-2 (compatible hypovirulent treatment inoculum) and the canker inciting strain MC-2. When isolate 2-13 (incompatible hypovirulent treatment inoculum) was paired with MC-2, hypovirus transmission never occurred despite numerous in vitro attempts. Double (1982) also noted that allelic asymmetry might be overcome more readily in vivo than in vitro. The specific vic genes responsible for separating the study isolates vegetatively did not completely prevent anastomosis.

The rate of canker expansion appeared to be related to the acquisition of hypovirus. In general, this was true as cankers that were exposed to compatible inoculum expanded significantly less than those treated with incompatible inoculum, in wounded treatments. For the non-invasive treatments, cankers exposed to compatible inoculum also expanded less than cankers treated with incompatible inoculum, although not significantly. Overall, $27 \%$ of all bark plugs recovered from the wounded incompatible treatments yielded orange-hypovirulent isolates, yet canker expansion was not reduced as with the compatible inoculum treatments. These orange-hypovirulent isolates cannot be attributed to propagules from hypovirulent-compatible cankers since separate, sterile bone marrow instruments were used to sample cankers in each treatment. While anastomosis and subsequent hypovirus acquisition occurred in the incompatible treatments, one explanation for the continued canker expansion might be that hypovirus 
titer was not at an adequate level to influence the processes that control the growth of the hyphae in bark. A further explanation may rest with the manner in which the samples and data were collected. Some cankers expanded irregularly yet the samples and canker size data were analyzed as if the cankers expanded elliptically. Limited expansion may have occurred in areas where hypoviruses were recovered but not in areas where bark invasion continued. While bark plugs were removed from specific locations in each canker, no association was made in the statistical analyses to link hypovirus recovery to areas of a canker that were actively expanding or had ceased growth.

Significantly more orange-hypovirulent isolates were recovered from areas of cankers that developed after hypovirus treatment (canker margins), among all treatments, compared to areas that developed prior to treatment. Conversely, the orange-virulent canker inciting isolates were isolated more frequently from the areas of cankers that existed prior to hypovirus treatment. If the most actively growing hyphae are in the outer portions of the expanding cankers then hypoviruses presumably are spread into these areas as the underlying fungal thallus expands. Jones (pers. com.) found in cultural studies that hyphae formed one-to-six weeks prior to hypovirus challenge did not acquire hypoviruses; only hyphae formed subsequent to challenge became hypovirulent. Therefore, Jones contends that the term "conversion" is inappropriate and suggests that hyphae that exist prior to exposure to hypoviruses remain virulent and it is only the subsequent growth that accounts for hypovirus expression. Jones concludes that when cankers yield hypovirulent isolates, they do so as a result of hypovirus replication that has occurred in the actively growing hyphae formed subsequent to hypovirus infection. 
Throughout this study, orange-virulent isolates and orange-hypovirulent isolates were isolated routinely from all cankers, yet no canker yielded only orange-hypovirulent isolates. These findings are consistent with Jones (pers. com.) and with a study at West Salem, Wisconsin. From 1992 through 1997, cankers at a West Salem site were treated with hypoviruses and fungal isolations were made annually from these cankers. Isolation results showed that treated cankers seldom yield hypovirulent isolates solely. Generally, virulent isolates are commonly isolated from these cankers when bark samples are cultured (Cummings-Carlson et al., 1998). Results from these studies indicate that while hypovirus-containing isolates are recovered from hypovirus treated cankers, acquisition does not proliferate throughout the entire canker thallus, regardless of vegetative compatibility types.

Presumably, for the phenomenon of hypovirulence to be an effective biological control, hypoviruses need to be transmitted into conidia efficiently and at high rates. Therefore, inoculum production also was evaluated during this study as a further measure of the success of canker treatment. Unfortunately, hypovirulent conidia were not produced in significant numbers. Most pycnidia that were sampled yielded virulent asexual spores even though the underlying thallus was mostly hypovirulent. This finding also was noted in a field study conducted by Shain and Miller (1992), where the rate and movement patterns of hypovirulent agents were studied after treatment of virulent cankers. They found that mycelium in bark was converted, whereas conidia formed in stromata above these areas were not. Prior to the initiation of my field experiment, single spores of the two brown-hypovirulent treatment isolates (80-2 and 2-13) were assessed for cultural morphology. These isolates yielded $70 \%$ and $96 \%$ hypovirulent conidia, 
respectively. It is unknown whether these isolates produce comparable numbers of hypovirulent conidia when grown in bark. Both isolates were initiated in study trees at the onset of the experiment, but neither isolate sporulated in vivo. Likewise, there were no preliminary laboratory experiments to determine whether the canker-inciting MC-2 isolate produces high or low numbers of hypovirulent conidia when it is hypovirus infected by the vegetatively compatible treatment isolate.

During the course of this study, many bark plugs yielded sectors containing various combinations of virulent / hypovirulent isolates. The sectoring phenomenon observed during this study also was observed by Hobbins (1985) and Balbalian (1998). Sectored isolates are those that have more than one strain of $C$. parasitica growing from the same bark plug. The most plausible explanation as to why sectored isolates occur is that the bark plugs actually may be colonized by different strains of $C$. parasitica (Kuhlman, 1982). The occurrence of sectored isolates probably would not have been noted if the brown-pigmented treatment isolate had not been used in this experiment. Also, when isolates were subcultured to PDA, there were fewer sectored isolates than were noted on GYE/A bark plug cultures. Undoubtedly, subculturing eliminated some sector components.

When sectors were observed, the orange-hypovirulent sectored isolates were most often associated with wounded cankers, regardless of whether the treatment inoculum was compatible or incompatible with the canker-inciting strain. Sectored isolates that included the brown-pigmented treatment inoculum were most commonly associated with the scratch-wounded treatments. The scratch wounds may have created more microenvironments in which the treatment inoculum had a better opportunity to become 
established in the bark than the punch wounds. Similarly, scratch wounded cankers also were responsible for significantly better survival of the treatment inoculum in the bark plug isolations. When cankers were treated with incompatible inoculum, most of the sectored isolates that were associated with the brown-hypovirulent treatment inoculum included an orange-virulent sector, regardless of treatment. Since the virulent canker inciting strain was less likely to anastomose with the incompatible brown-hypovirulent treatment inoculum, this would be an expected outcome if the cultured bark plug had been colonized by both strains. Therefore, the phenomenon of vegetative incompatibility probably played a role in the sectoring process.

While sectoring in $C$. parasitica has been reported previously, this study is the first to report different levels of pigmentation in the orange-hypovirulent isolates recovered from bark plugs. Some isolates recovered from bark samples had typical European hypovirus morphology; an orange-pigmented colony center with abundant white, aerial hyphae around the margin (Figure 20). This isolate type was referred to as "high-pigmented". Other isolates recovered from bark had much less orange-pigment and reduced growth and limited aerial hyphae. This morphology type was referred to as "low-pigmented" (Figure 20). The "high" and "low" pigmentation phenomenon was neither observed among the brown-hypovirulent treatment inoculum isolates that were recovered nor was pigmentation associated with any particular treatment type. The pigment type could be stabilized when either the high or low-pigmented mass isolates were single spored. That is, single sporings of isolates from "high" pigmented single spore colonies yielded only "high" pigmented colonies. Likewise, single sporing "low" pigmented single spore colonies yielded only "low" pigmented colonies. Virulent 
colonies were not evident in any single spore attempts. This was unusual because virulent colonies often can be identified after single sporing hypovirus-infected isolates. The dsRNA analyses illustrated that both the "high" and "low"-pigmented isolates are hypovirus containing. Yet, in a preliminary virulence test, the low-pigmented isolate type was less pathogenic than the high-pigmented isolate (Figure 22). Further testing is required to determine the cause of the high and low pigmentation and whether the pigment stability noted in this study occurred because of a nuclear condition such as heterokaryosis.

Double (1982) and Balbalian (1998) concluded from their studies that other methods for introduction of hypovirulent inoculum need to be developed and tested. This study provided further insight to the variables associated with successful treatment. Clearly, this experiment showed that wounding is paramount for hypovirus acquisition. Previous field studies routinely have relied on punch-wounding cankers at their margin as the principal treatment method (Grente and Berthelay-Sauret, 1978; Jaynes and Elliston, 1980; Shain and Miller, 1992). This study confirms that the punch-wounding method is an effective way to deliver hypovirulent inoculum. Also, covering the cankers after treatment provides a method to preserve treatment inoculum, although successful establishment of hypoviruses do not appear dependent on the canker coverings, at least not during the time frame in which this study occurred.

Future research based on these experiments might further refine the treatments used in this study and should focus on the methods where the most successful hypovirus acquisition occurred. The season of hypovirus application could be considered. Even though cankers treated in this study were initiated during the host's active growing 
season, hypovirus treatments were not introduced until August $23^{\text {rd }}, 2002$, toward the end of the period of the most active canker expansion. Treating cankers at the onset of the growing season is another variable worth examining and may result in better hypovirus acquisition during a more active period of hyphal growth. Control treatments where cankers are treated with water agar could be added to provide comparison to hypovirus treatments. This would be especially useful for vegetatively incompatible combinations where growth did not appear to be slowed but hypovirus transmission occurred. As mentioned earlier, the possibility exists that there may be a threshold of hypovirulent hyphae needed to halt growth. Perhaps the vegetatively incompatible treatments did offer a level of control, but since there were no corresponding treatments that utilized virulent isolates, this could not be measured. Further, this was a single season experiment; the fate of these cankers after a second growing season may have provided different conclusions. Unfortunately, the health of the trees due to endemic blight precluded collecting a second season's data.

The limited production of hypovirulent inoculum is an issue that also needs to be addressed. There may be procedures that can be developed which would increase hypovirulent spore production following treatment. The possibility exists that the survival of the treatment inoculum that was noted in the scratch wounded and covered treatments could result in increased hypovirulent inoculum production over longer time periods. Additional studies comparing isolates where the vic gene relationships are known could be useful in further defining better hypovirus delivery techniques. By knowing the vic gene relationships among isolates, studies could be designed where the 
degree of incompatibility between isolates is known precisely, providing a better measure of the role of vic genes and the type of treatments.

In conclusion, this study has clearly demonstrated that wounding is a critical prerequisite when treating cankers with inoculum containing hypovirulence agents. Further, of all the procedures that were tested in this study, punch-wounding cankers prior to hypovirulent treatment remains the best procedure to introduce hypoviruses for biological control of $C$. parasitica. Also, covering cankers following treatment with hypovirulent inoculum doesn't seem to be critical to hypovirus transmission. This study confirms that the vegetative compatibility relationship between the canker-inciting strain and hypovirulent treatment inoculum is an important prerequisite for the success of biological control. 
Summary

1. When cankers were treated with incompatible inoculum, canker diameter increased significantly more than when cankers were treated with compatible inoculum, regardless of treatment. Also, there was significantly less expansion in wounded cankers than non-wounded cankers.

2. Hypovirus transmission occurred more frequently when cankers were treated with compatible hypovirulent inoculum, regardless of treatment. The best transmission was detected when cankers were wounded, especially when they were punch treated. Painting cankers with hypovirulent inoculum, without wounding, resulted in significantly less hypovirus transmission than when cankers were wounded. Also, there was a significantly higher frequency of orange-hypovirulent isolates recovered from post-treatment areas of cankers than pre-treatment areas: the converse is also true of the canker inciting orange-virulent isolate (MC-2). When recovery of the MC-2 strain was high, there was always a corresponding decrease in recovery of orange-hypovirulent isolates and brown-hypovirulent treatment isolates.

3. Covering cankers with the absorbent pads for an 18-day period following treatment significantly promoted the survival of the brown hypovirulent inoculum source, but did not enhance the transmission of hypoviruses. The best survival of treatment inoculum occurred within the cankers when they were scratch wounded, which is opposite to the recovery of orange-hypovirulent isolates. Treatments with incompatible hypovirulent inoculum yielded significantly more isolations of the brown hypovirulent treatment inoculum than did treatments with compatible hypovirulent inoculum.

4. When orange-hypovirulent isolates were recovered from bark plugs, they expressed high and low levels of pigmentation. The low-pigmented isolates were less pathogenic than the high-pigmented isolates in an apple virulence model, which were comparably pathogenic to the canker inciting virulent strains. Both pigmented isolate types contained dsRNA.

5. Single spore colonies from bark pycnidia resulted in OV spores being recovered in higher frequencies than other spore types regardless of treatment. When hypovirulent spores were recovered, although at very low frequencies, they were from treatments that involved wounding.

6. Sectoring from bark plug colonies was noted more during the initial culturing of the bark plugs on GYE/A media. The most common sectored isolate was the $\mathrm{OV} / \mathrm{BH}$ type and was found mostly in the incompatible wounded treatments. When sectors involved $\mathrm{OH}$ isolates, they were recovered more frequently from wounded treatments. 


\section{References}

Anagnostakis, S.L. 1977. Vegetative compatibility in Endothia parasitica. Exp. Mycol. 1:306-316.

Anagnostakis, S.L., and P.E. Waggoner. 1981. Hypovirulence, vegetative incompatibility, and the growth of cankers of chestnut blight. Phytopathology 71:1198-1202.

Anderson, P.J. and H.W. Anderson. 1912. The chestnut blight fungus and a related saprophyte. Phytopathology 2:204-212.

Balbalian, C.J. 1998. The influence of vegetative incompatibility genes on the transmission of hypoviruses between strains of Cryphonectria parasitica. Master's thesis. West Virginia University. Morgantown. 101 pp.

Barr, M.E. 1978. The Diaporthiales in North America. Mycologia Mem. 7. J. Cramer. Lehre, Germany. 232 pp.

Biraghi, A. 1953. Possible active resistance to Endothia parasitica in Castanea sativa. pgs. 643-645 in: Rep. Congr. Int. Union For. Res. Org. $11^{\text {th }}$.

Braun, E. Lucy. 1950. Deciduous Forests of Eastern North America. McGraw-Hill Book Co. Inc., New York. 596 pgs.

Cortesi, P. and M.G. Milgroom. 1998. Genetics of vegetative incompatibility in Cryphonectria parasitica. Appl. Environ. Microbiol. 64:2988-2994.

Cummings-Carlson, J., D.W. Fulbright, W.L. MacDonald, and M.G. Milgroom. 1998. West Salem: a research update. J. Amer. Chestnut Found. 12:24-26.

Double, M.L. 1982. The ability of hypovirulent isolates and mixtures of hypovirulent isolates to control artificially established virulent cankers. pgs. 145-152. in: Proc. USDA Forest Service American Chestnut Coop. Meet., H.C. Smith and W.L. MacDonald, eds. W. Va. Univ. Books, Morgantown, WV. 229 pgs.

Elliston, J.E. 1982. Hypovirulence in Endothia parasitica and suggested procedures for its detection and analysis. pgs. 1-13 in: Proc. USDA Forest Service American Chestnut Coop. Meet., H.C. Smith and W.L. MacDonald, eds. W.Va. Univ. Books, Morgantown, WV. 229 pgs.

Fulbright, D.W., W.H. Weidlich, K.Z. Haufler, C.S. Thomas, and C.P. Paul. 1983. Chestnut blight and recovering American chestnut trees in Michigan. Can. J. Bot. 61:3164-3171. 
Grente, J., and S. Berthelay-Suaret. 1978. Biological control of chestnut blight in France. Pages 30-34 in: Proc. Am. Chestnut Symposium., W.L. MacDonald, F.C. Cech, J.Luchok, and C. Smith, eds. W.Va. Univ. Books, Morgantown.

Griffin, G.J. 1986. Chestnut blight and its control. Hortic. Rev. 8:291-336.

Heald, F.D. and M.W. Gardner. 1913. The relative prevalence of pycnospores and ascospores of the chestnut blight fungus during winter. Phytopathology 3:296305.

Hillman, B.I., D.W. Fulbright, D.C. Nuss, and N.K. vanAlfen. 1995. pgs. 261264 in: Virus Taxonomy, Classification and Nomenclature of Viruses. Sixth Report of the International Committee on Taxonomy of Viruses. F.A. Murphy, C.M. Fauquet, D.H.L. Bishop, S.A. Ghabrial, A.W. Jarvis, G.P. Martelli, M.A. Mayo, and M.D. Summers (eds.) Springer-Verlag, Wein, New York.

Hobbins, D.L. 1985. Interactions between the thallus of virulent Endothia parasitica cankers and sources of virulent and hypovirulent inoculum on American chestnut. Master's thesis. West Virginia University. Morgantown. $104 \mathrm{pp}$.

Huber, D.H. 1996. Genetic analysis of vegetative incompatibility polymorphisms and horizontal transmission in the chestnut blight fungus Cryphonectria parastica. Ph.D. diss., Michigan State University. East Lansing. 204 pp.

Jaynes, R.A., and N.K. DePalma. 1982. Attempts to control chestnut blight with slurry and conidial sprays of hypovirulent strains. pgs. 128-133. in: Proc. USDA Forest Service American Chestnut Coop. Meet., H.C. Smith and W.L. MacDonald, eds. W. Va. Univ. Books, Morgantown, WV. 229 pgs.

Jaynes, R.A., and J.E. Elliston. 1980. Pathogenicity and canker control by mixtures of hypovirulent strains of Endothia parasitica in American chestnut. Phytopathology 70:453-456.

Jaynes, R.A. and J.E. Elliston. 1982. Hypovirulent isolates of Endothia parasitica associated with large American chestnut trees. Plant Dis. 66:769-772.

Kuhlman, E.G. 1978. The devastation of American chestnut by blight. p. 1-3. in: Proc. Am. Chestnut Symposium., W.L. MacDonald, F.C. Cech, J.Luchok, and C. Smith, eds. W.Va. Univ. Books, Morgantown. 122 pgs.

Kuhlman, E.G. 1982. Vegetative compatibility and hypovirulence conversion in Endothia parasitica: state of the art. pgs. 210-217. in: Proc. USDA Forest Service American Chestnut Coop. Meet., H.C. Smith and W.L. MacDonald, eds. W. Va. Univ. Books, Morgantown, WV. 229 pgs. 
Liu, Y.C. and M.G. Milgroom. 1996. Correlation between hypovirus transmission and the number of vegetative incompatibility (vic) genes different among isolates from a natural population of Cryphonectria parasitica. Phytopathology 86:79-86

MacDonald, W.L., D.F. Hindal, and W.J. Kaczmarczyk. 1982. Summary of Endothia parasitica hypovirulence research at West Virginia University. pgs. 1823. in: Proc. USDA Forest Service American Chestnut Coop. Meet., H.C. Smith and W.L MacDonald, eds. W.Va. Univ. Books, Morgantown, WV. 229 pgs.

MacDonald, W.L. and D.W. Fulbright. 1991. Biological control of chestnut blight: use and limitations of transmissible hypovirulence. Plant Dis. 75:656-661.

MacDonald, W. L. and M. L. Double. 1978. Frequency of vegetative-compatibility types of Endothia parasitica in two areas of West Virginia. pgs. 103-105. in: Proc. Am. Chestnut Symposium. W.L. MacDonald, F.C. Cech, J.Luchok, and C. Smith, eds. W.Va. Univ. Books, Morgantown.

Merkel, H.W. 1905. A deadly fungus on the American chestnut. N.Y. Zool. Soc. $10^{\text {th }}$ Ann. Rep. p. 97-103.

Milgroom, M.G. and P. Cortesi. 2004. Biological control of chestnut blight with hypovirulence: a critical analysis. Annu. Rev. Phytopathol. 42:311-338.

Mittempergher, L. 1978. The present status of chestnut blight in Italy. pgs. 34-37 in: Proc. Am. Chestnut Symposium. W.L. MacDonald, F.C. Cech, J.Luchok, and C. Smith, eds. W.Va. Univ. Books, Morgantown.

Morris, T.J. and J.A Dodds. 1979. Isolation and analysis of double-stranded RNA from virus-infected plant and fungal tissue. Phytopathology 69:854-858.

Powell, W.A. 1995. Vegetative incompatibility and mycelial death of Cryphonectria parasitica detected with a $\mathrm{pH}$ indicator. Mycologia 87:738-741.

Russin, J.S., L. Shain, and G.L. Nordin. 1984. Insects as carriers of virulent and cytoplasmic hypovirulent isolates of the chestnut blight fungus. J. Econ. Entomol. 77:838-846.

Scharf, C.S., and N.K. DePalma. 1981. Birds and mammals as vectors of the chestnut blight fungus (Endothia parasitica). Can. J. Zool. 59:1647-1650.

Shain, L. and J.B. Miller. 1992. Movement of cytoplasmic hypovirulence agents in chestnut blight cankers. Can. J. Bot. 70:557-561.

Shear, C.L., N.E. Stevens, and R.J. Tiller. 1917. Endothia parasitica and related species. USDA Bull. 380. 82 p. 
Appendices

Appendix A: Media Used

\section{Glucose Yeast Extract with Antibiotics}

(GYE/A)

$\begin{array}{lc}\text { Glucose } & 10.0 \mathrm{~g} \\ \text { Yeast Extract } & 2.0 \mathrm{~g} \\ \mathrm{KH}_{2} \mathrm{PO}_{4} & 1.0 \mathrm{~g} \\ \mathrm{MgSO}_{4} & 0.5 \mathrm{~g} \\ \text { Thiamine } & 50 \mu \mathrm{g} \\ \text { Biotin } & 10 \mu \mathrm{g} \\ \text { Microelements } & \text { Trace amount } \\ \text { (Iron, Manganese, Zinc) } & \\ \text { Agar } & 20.0 \mathrm{~g} \\ \text { Distilled Water } & 1000.0 \mathrm{ml} \\ \text { Tetracycline Hydrochloride } & 100 \mathrm{mg} \\ \text { Streptomycine Sulfate } & 10 \mathrm{mg}\end{array}$

Antibiotics were added to cooled media after autoclaving.

\section{Potato Dextrose Agar \\ (PDA)}

PDA (Difco)

Methionine

Biotin

Distilled Water
$39.0 \mathrm{~g}$

$0.1 \mathrm{~g}$

$10 \mu \mathrm{g}$ $1000.0 \mathrm{ml}$

For dsRNA extraction, agar plugs were inoculated onto sterile cellophane, overlaid on surface of PDA. 


\section{Bromcresol Green}

(BCG)

PDA

Malt Extract

Yeast Extract

Tannic Acid

Bromcresole Green

Distilled Water

Agar

Tween-20
$24.0 \mathrm{~g}$

$7.0 \mathrm{~g}$

$2.0 \mathrm{~g}$

$0.8 \mathrm{~g}$

$50.0 \mathrm{mg}$

$1000.0 \mathrm{ml}$

$15.0 \mathrm{~g}$

12 drops

\section{Water Agar}

Granulated Agar

$28.0 \mathrm{~g}$

Tap Water $1200.0 \mathrm{ml}$

\section{Peptone Blanks}

Bacto-Peptone (Difco) $1.0 \mathrm{~g}$

Distilled Water $\quad 1000.0 \mathrm{ml}$ 


\section{Appendix B: dsRNA Extraction Protocol}

Cellophane (Flexal Corp.) is cut to the diameter of 100x15 mm petri plates (Fisherbrand Scientific), added to a glass petri dish containing tap water, covered, and autoclaved for 24 minutes at $15 \mathrm{psi}$. The cellophane is then aseptically transferred to PDA plates amended with tetracycline hydrochloride and streptomycin sulfate. Agar plugs containing mycelium of test isolates are replicated on 5 plates. Plates are incubated at $20^{\circ} \mathrm{C}$ for 7-10 days. The mycelium is then scraped from the cellophane into a cold mortar, immersed in liquid nitrogen and ground to a fine powder.

The mycelial powder is added to a $30 \mathrm{ml}$ polyallomer screw cap test tube, to which is added: $10 \mathrm{ml} 2 \mathrm{X}$ STE (containing $20 \%$ sodium dodecyl sulfate), $11 \mathrm{ml}$ phenol (containing $0.1 \%$ 8-hydroxyquinoline) and $8 \mathrm{ml}$ chloroform / isoamyl alcohol (24:1). Tubes are capped, covered with ice and placed on a rotary shaker ( $\sim 100 \mathrm{rpm})$ for 30-45 minutes. After shaking, the tubes are centrifuged for 30 minutes at $8,000 \mathrm{rpm}$ at $0-4^{\circ} \mathrm{C}$. The aqueous phase is collected in a $25 \mathrm{ml}$ graduated cylinder and the volume brought up to $20 \mathrm{ml}$ with $1 \mathrm{X}$ STE and $95 \% \mathrm{EtOH}$ added to a final concentration of $15 \%$.

Twelve grams of chromatographic cellulose powder (Whatman CF-11 cellulose) is equilibrated with $200 \mathrm{ml} \mathrm{STE}: 15 \% \mathrm{EtOH}$. The cellulose solution is mixed continuously and $25 \mathrm{ml}$ is added to fritted glass columns and allowed to drain. The nucleic acid sample is then applied to the column and washed with 80-100 ml STE:15\% EtOH. Bromphenol blue solution is added to the surface of the CF-11 column (3-5 drops) then nucleic acids are eluted with $11 \mathrm{ml} 1 \mathrm{X}$ STE (no alcohol). Elute is collected in a $30 \mathrm{ml}$ glass Corex tube.

Eighteen $\mathrm{ml}$ of $95 \% \mathrm{EtOH}$ and 9 drops of $3 \mathrm{M}$ sodium acetate are added to each tube. The tubes are covered with parafilm and the contents mixed thoroughly. The samples are stored at $-20^{\circ} \mathrm{C}$ overnight.

Upon removal from the freezer, the tubes are centrifuged at $8000 \mathrm{rpm}$ for 30 minutes. The supernatant is decanted and the excess alcohol is wiped from the tubes along the line of centrifugation with kimwipes. The dsRNA precipitate is resuspended with $1 \mathrm{ml}$ of the resuspension buffer and vortexed for 30 seconds.

Traces of DNA are removed from the nucleic acid sample by adding $100 \mu 10.5 \mathrm{M}$ $\mathrm{MgCl}_{2}$ and 20 $\mu$ DNAse (Promega RQ1 RNAse-free DNAse) for 60 minutes. Two $\mathrm{ml}$ of cold $95 \% \mathrm{EtOH}$ and 1 drop of sodium acetate are then added to each tube. The solution is vortexed and the tubes are stored at $-20^{\circ} \mathrm{C}$ for 2 hours.

Samples are centrifuged at $14,000 \mathrm{rpm}$ and alcohol is decanted. The remaining pellet is dried and resuspended in $15 \mu 1$ resuspension buffer.

An agarose gel stained with ethidium bromide is immersed in 1L of $1 \mathrm{X}$ TBE in an electrophoresis tank. Each well is loaded with $5-10 \mu \mathrm{l}$ of sample. The gel is electrophoresed for 2 hours at $100 \mathrm{mV}$. Gels are examined under UV light $(250 \mathrm{~nm})$ for florescent bands of dsRNA and photographed with Genesnap software (Syngene ${ }^{\circledR}$ ). 


\section{Buffers for dsRNA extractions}

10X STE:

$0.5 \mathrm{M}$ Tris $\quad 61.0 \mathrm{~g}$

$0.1 \mathrm{M}$ Sodium chloride $\quad 58.0 \mathrm{~g}$

0.001M Disodium EDTA $\quad 3.7 \mathrm{~g}$

Distilled Water $\quad 1000.0 \mathrm{ml}$

$\mathrm{pH}$ adjusted to 6.8 with glacial acetic acid

10X TBE

Tris

Boric Acid

$54.5 \mathrm{~g}$

Disodium EDTA

Distilled Water

$27.8 \mathrm{~g}$

$1.9 \mathrm{~g}$

$1000.0 \mathrm{ml}$

\section{Bromcresol Blue Solution}

Bromcresol Blue

$50.0 \mathrm{mg}$

Sucrose

$25.0 \mathrm{~g}$

Distilled Water

$50.0 \mathrm{ml}$

\section{$\underline{\text { Resuspension Buffer }}$}

10X TBE

$2.0 \mathrm{ml}$

Sucrose

$4.0 \mathrm{~g}$

Distilled Water

$38.0 \mathrm{ml}$ 
Appendix C: Sample ANOVA Table

$\underline{\text { Source }}$

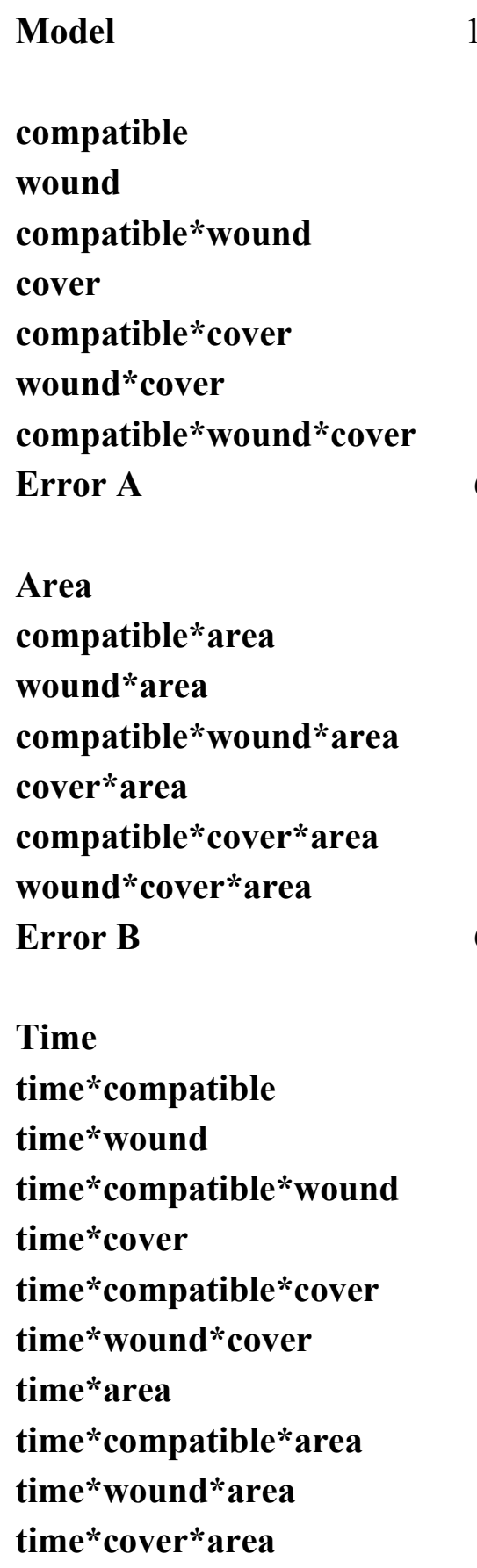

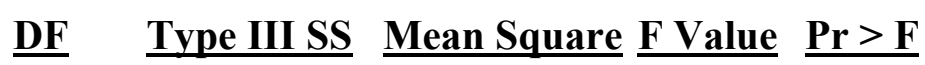

$\begin{array}{ccccc}158 & 578.913316 & 3.6640083 & 4.8 & <0.0001 \\ 1 & 108.6807347 & 108.6807347 & 52.68 & <0.0001 \\ 2 & 130.4195566 & 65.2097783 & 31.61 & <0.0001 \\ 2 & 42.0261353 & 21.0130677 & 10.19 & 0.0002 \\ 1 & 0.047172 & 0.047172 & 0.02 & 0.8803 \\ 1 & 0.465246 & 0.465246 & 0.23 & 0.6366 \\ 2 & 4.6061053 & 2.3030527 & 1.12 & 0.3342 \\ 2 & 3.3748913 & 1.6874457 & 0.82 & 0.4462 \\ 60 & 123.7810543 & 2.0630176 & 2.7 & <0.0001\end{array}$

$\begin{array}{ccccc}1 & 38.62224244 & 38.62224244 & 30.09 & <0.0001 \\ 1 & 1.24038665 & 1.24038665 & 0.97 & 0.3294 \\ 2 & 1.09326383 & 0.54663192 & 0.43 & 0.6551 \\ 2 & 17.96480774 & 8.98240387 & 7 & 0.0018 \\ 1 & 3.92734193 & 3.92734193 & 3.06 & 0.0852 \\ 1 & 4.71210103 & 4.71210103 & 3.67 & 0.06 \\ 2 & 2.95324102 & 1.47662051 & 1.15 & 0.3232 \\ 62 & 79.5798783 & 1.2835464 & 1.68 & 0.007\end{array}$

$\begin{array}{ccccc}1 & 0.1701831 & 0.1701831 & 0.22 & 0.6376 \\ 1 & 0.1193 & 0.1193 & 0.16 & 0.6933 \\ 2 & 2.9057003 & 1.4528501 & 1.9 & 0.1533 \\ 2 & 5.6966509 & 2.8483254 & 3.73 & 0.0266 \\ 1 & 0.8499034 & 0.8499034 & 1.11 & 0.2933 \\ 1 & 0.0124829 & 0.0124829 & 0.02 & 0.8984 \\ 2 & 2.2870703 & 1.1435352 & 1.5 & 0.2274 \\ 1 & 1.528294 & 1.528294 & 2 & 0.1595 \\ 1 & 0.8834338 & 0.8834338 & 1.16 & 0.284 \\ 2 & 0.5367503 & 0.2683751 & 0.35 & 0.7043 \\ 1 & 0.4293885 & 0.4293885 & 0.56 & 0.4546\end{array}$

Error

$129 \quad 98.4782673 \quad 0.7633974$ 